Review Article

\title{
Incorporating Colour Information for Computer-Aided Diagnosis of Melanoma from Dermoscopy Images: A Retrospective Survey and Critical Analysis
}

\author{
Ali Madooei and Mark S. Drew \\ School of Computing Science, Simon Fraser University, Burnaby, BC, Canada \\ Correspondence should be addressed to Ali Madooei; amadooei@cs.sfu.ca
}

Received 25 August 2016; Revised 5 November 2016; Accepted 23 November 2016

Academic Editor: D. L. Wilson

Copyright (C) 2016 A. Madooei and M. S. Drew. This is an open access article distributed under the Creative Commons Attribution License, which permits unrestricted use, distribution, and reproduction in any medium, provided the original work is properly cited.

\begin{abstract}
Cutaneous melanoma is the most life-threatening form of skin cancer. Although advanced melanoma is often considered as incurable, if detected and excised early, the prognosis is promising. Today, clinicians use computer vision in an increasing number of applications to aid early detection of melanoma through dermatological image analysis (dermoscopy images, in particular). Colour assessment is essential for the clinical diagnosis of skin cancers. Due to this diagnostic importance, many studies have either focused on or employed colour features as a constituent part of their skin lesion analysis systems. These studies range from using low-level colour features, such as simple statistical measures of colours occurring in the lesion, to availing themselves of high-level semantic features such as the presence of blue-white veil, globules, or colour variegation in the lesion. This paper provides a retrospective survey and critical analysis of contributions in this research direction.
\end{abstract}

\section{Introduction}

Image-based computer-aided diagnosis systems have significant potential for screening and early detection of melanoma. At present, digital dermoscopic images seem to be the most promising source for automatic or computer-aided melanoma diagnosis [1]. Automatic dermoscopy image analysis is mainly concerned with two tasks: (i) identifying dermoscopic features in each image and (ii) associating those features with a diagnosis (image classification of skin lesions). Both tasks are examples of visual recognition that itself is a classical problem of computer vision. The organization of a computer vision system is highly application-dependent. For automatic dermoscopy image analysis, a typical system pipeline involves preprocessing, lesion segmentation, feature extraction, and classification. These steps are briefly described next. For a detailed survey, the interested reader is referred to $[2,3]$.

(i) Preprocessing used for dermoscopy images is of many kinds, including but not limited to image enhancement (such as colour correction, shading removal, and contrast adjustment), colour space transformation, and removal of artifacts (such as hairs, ruler markings, air bubbles, black frames, and ink markings). Among these, previous efforts have mostly been focused on the development of hair removal algorithms.

(ii) Lesion segmentation involves isolating skin lesions from normal skin surrounding it. It is important for two reasons: Firstly, the lesion boundary provides important information for accurate diagnosis. For example, border asymmetry or irregularity can signal malignancy. Secondly, it is a common practice to perform feature extraction for the lesion area only, masking the healthy skin surrounding it. Therefore, representativeness of image features depends on the accuracy of the segmentation. Lesion segmentation is one of the most studied topics. It poses a challenge for several reasons, including low contrast between the lesion and its background and the presence of artifacts. 
(iii) Feature extraction involves detection, description, and representation of image features. The concept of image feature is very general but, in the context of dermoscopy image analysis, it can be defined as computing abstractions of information contained within the image that is relevant for diagnosis. This abstraction can be made at different levels (local versus global, low-level versus high-level) depending on how subsequent algorithms operate. For example, primitive or low-level features such as the distribution of image intensity, texture, or colour may be used for solving the computational task related to the diagnosis of a medical condition. A common trend in recent years is in developing high-level semantic (clinically meaningful) visual features that can be used by both clinicians and computer programs for computer-assisted diagnosis.

(iv) Classification is usually the final step. The output of lesion classification depends on the application but it is often an estimate of the probability of malignancy. To this aim, a typical approach would be based on the classical paradigm of supervised machine learning that requires training images. Classification might be preceded by feature selection which is the process of selecting a subset of relevant features for use in model construction. In many cases, the derivation of high-level semantic features also requires the use of classification (or other machine learning techniques).

Colour features are among the most widely used features in image analysis tasks. Colour has been arguably the most widely used feature in dermoscopy image analysis as well. This is not a surprise since colour is an essential part of most clinical methods for screening of skin lesions. The presence of multiple colours with an irregular distribution highly suggests malignancy. Among dermoscopic features of melanoma, colour has a substantial role too. The presence of certain shades of blue in a lesion implies the possible diagnosis of melanoma more so than a lesion manifesting only different shades of brown. Thus, the correct clinical diagnosis of a pigmented skin lesion requires accurate evaluation of its colouration in terms of its shades and distributions. This paper is aimed at reviewing the literature on incorporating colour features for computer-aided diagnosis through dermoscopy image analysis.

The studies cited in this review were retrieved through a careful literature search using several databases, namely, IEEE Xplore (http://ieeexplore.ieee.org/), Springer Link (http://link.springer.com/), Science Direct (http://www .sciencedirect.com/), Wiley Online Library (http://onlinelibrary.wiley.com/), Web of Science (http://webofscience .com/), PubMed (http://www.ncbi.nlm.nih.gov/pubmed), and Google Scholar (https://scholar.google.com/). One of the challenges of finding relevant literature, as it is with cross-disciplinary research, is to survey a wide array of topic areas from clinical practice to scientific research that includes specialized journals to multidisciplinary conferences. The initial search strategy included keyword search in combination with category filtering and retrieved more than 1500 articles. These articles were archived using Zotero, a web browser plug-in for gathering and organizing research resources. Using Zotero, duplicated results were removed. Moreover, through analysis of articles' titles and keywords, all results pertaining to the following categories were manually removed:

(i) Studies that used imaging modalities other than dermoscopy or clinical photography, including confocal microscopy, histopathology, laser Doppler, MRI, multiphoton laser tomography, ultrasound, near infrared, (any type of) spectroscopy, fluorescence imaging, stereo imaging, and multi- or hyperspectral imaging.

(ii) Studies that did not focus on computer-aided diagnosis through image analysis. For example, clinical trials, case studies, or studies on genome, biomarkers, bioprofile, and proteins were all removed.

(iii) Studies which were not aimed at diagnosis of skin cancer. For example, topics of "wound healing evaluation," "assessment of burn scars," "erythema measurement," and "detection of psoriasis" were removed.

The initial retrieval set was reduced to about 600 articles at this stage. This reduced set was carefully reviewed to further identify those studies using colour features for computeraided diagnosis through dermoscopy image analysis. At the end, more than 100 articles (published from 1989 to 2015 in more than 20 different publications) were selected. This final set is critically reviewed in the following sections.

\section{Clinical Background}

Dermoscopy (a.k.a. epiluminescence microscopy, dermatoscopy, or amplified surface microscopy) uses a handheld microscope called a dermatoscope (or dermoscope) for in vivo examination of skin lesions. It can be used to differentiate most lesions of the skin from melanoma [4]. In the hands of experienced users, dermoscopy can improve the clinicians' diagnostic accuracy by $10 \%$ to $30 \%$ (depending on the type of skin lesion) $[5,6]$ and increases the physicians' confidence in their clinical diagnoses $[7,8]$, thus significantly reducing unneeded biopsies [9].

The dermoscopic diagnosis of pigmented skin lesions is based on various analytic approaches or algorithms that have been set forth in the last few decades. The common denominators of all these diagnostic methods are particular dermoscopic features (such as colours and patterns) that represent the backbone for the morphologic analysis of pigmented skin lesions.

2.1. Dermoscopic Colours. Skin colour is the result of the interaction of light with skin pigments (melanin, hemoglobin, and keratin) and other materials (collagen, serum in crusts, etc.). Dermatoscope reveals a wider range of colours than those that can be seen with the naked eye. Common colours under dermatoscope include various shades of brown, blue, black, white, and red. There is more of these colours than those that meet the eye; colours can convey critical morphologic information. For instance, shades of blue 
might be caused by the presence of melanin pigment in the deeper layers of skin (deeper than expected) which itself can be a clue to invasive melanoma. Among other colours, shades of red can be associated with increased vascularization, dilatation of blood vessels, and bleeding within the lesion.

Melanin is the main chromophore in pigmented skin lesions. Melanin is black but, depending on how deeply and densely in the skin it is located, it might be perceived differently. If melanin is situated in the stratum corneum or immediately beneath it, the lesion would appear black on dermoscopy. Melanin at the dermoepidermal junction would be perceived as light brown to dark brown, depending on its density. Melanin in the dermis results in shades of grey to blue (when located in the deeper dermis).

The other important determinant of lesion colouration is hemoglobin in red blood cells which results in shades of pink and red (depending on the vascular volume). Poorly oxygenated blood might be perceived as purple, and congealed blood (thrombus) often appears as jet black. The stratum corneum, devoid of blood and melanin, appears yellow. Orange hues are due to serum present in erosions or superficial ulcerations. White colour is due to depigmentation, fibrosis, alterations in the collagen matrix, or keratin within cysts.

Benign lesions (usually) reveal only one or two colours whereas melanomas (frequently) reveal three or more colours. Thus, the number of colours present in a lesion can also help differentiate many benign nevi from melanomas. It is important, however, to appreciate that not all colours have the same impact on the diagnosis of melanoma. The presence of red, white, and/or blue-grey colours in a lesion implies the possible diagnosis of melanoma more so than a lesion manifesting only different shades of brown. Moreover, distribution of colours in melanoma are often focal, asymmetrical, and irregular. Thus, the correct clinical diagnosis of a pigmented skin lesion requires an accurate evaluation of its colouration (concerning the shades and the distribution of colours).

In addition to common colour names, dermatologists often use other descriptive terms to describe skin colouration. These include

(i) hyperpigmentation: skin colour that is darker than normal. This is usually associated with increased melanin (melanosis)

(ii) hypopigmentation: skin colour that is paler than normal but not completely white (often associated with loss of melanin).

(iii) erythema: skin redness due to increased blood supply. It might occur with any skin injury, infection, or inflammation. [10].

For a detailed survey, the interested reader is referred to

\section{Colour Moments}

Colour moments characterize colour distribution in an image. To this aim, first and second moments (mean and variance) are the most commonly used features, although third and fourth moments (skewness and kurtosis) are sometimes employed as well. Higher-order moments are usually not part of the colour moment feature set, because, to obtain a good estimate of their value, more data is required (also, they are more sensitive to noise). Instead, some authors include other statistical measures such as maximum, minimum, range, and entropy alongside colour moments. These simple statistical parameters are easy to compute and have been used extensively in dermoscopy image analysis literature [11-35].

Colour moments are usually calculated for each colour channel separately over a region or the entire image. They can be computed for any colour model. Some authors combined different colour spaces to enrich the colour moment feature set. Ganster et al. [30], for instance, employed the minimum, maximum, average, and the variance of the RGB and HSI colour channels as colour features.

Colour moments have been extensively used for image retrieval [36-39]. The main advantage of using colour moments is that there is no need to store the complete colour distribution [40]. This speeds up image retrieval since there are fewer features to compare. Also, colour moments are scaling and rotation invariant. On the downside, these features are most useful when they are calculated to represent a homogeneous region. They are not often enough to represent all the colour information of an image.

\section{Colour Histogram}

A colour histogram represents the distribution of the composition of colours in an image. It quantizes a colour space into different "bins" and counts the frequency of pixels belonging to each colour bin. In general, a histogram is a representation of the distribution of "continuous numerical data," like image intensities, where data is grouped into ranges (i.e., bins). When used as an image feature, a histogram is a vector with as many elements as the number of bins, and each element is the count of pixels that "fall" into the corresponding bin.

Colour histograms have been widely employed in dermoscopy image analysis literature. For instance, Xie et al. [26] used a multivariate histogram to capture the colour variegation of lesions. In their method, the RGB colour space was quantized into $16 \times 16 \times 16$ bins and the joint distribution of RGB data was represented via a 3D histogram. In addition, Xie et al. captured the colour differences between lesion and healthy skin surrounding it and used that as an image feature. To this aim, they computed the Euclidean distance between the histogram of lesion and the histogram of peripheral area around the lesion. The latter was carried out using $3 \mathrm{D}$ histograms with $4 \times 8 \times 8$ bins in CIE Luv colour space. Other notable studies include the works of Stanley et al. [41], Celebi et al. [11], Rahman and Bhattacharya [33], Situ et al. [42], Ruela et al. [12, 43], and Barata et al. [22, 24, 44].

The formation of a histogram involves various principles. For instance, one can choose to have fixed or variable bin width. One can use "hard assignment" where intensity values are assigned to the closest bin or "soft assignment" instead, where each pixel contributes to all bins based on the distance to all bin centers. For a colour histogram, there are other considerations. For example, one can choose to create a histogram for each colour channel and then concatenate these into one feature vector. This format is often called 
unidimensional or univariate histogram. Alternatively, one can choose to model the joint distribution of colour channels and create a large histogram that accounts for every combination of triple colour values. The latter is called a multidimensional or multivariate histogram. A colour histogram does not contain pixels' spatial information. The advantage is that since a histogram does not provide spatial information, it is rotation and translation invariant. The disadvantage is that two images with the same colour content but different spatial distribution of these colours are indistinguishable based solely on colour histogram comparisons.

\section{Colour Asymmetry}

Asymmetry is a feature inspired by the clinical diagnosis guidelines. In the $\mathrm{ABCD}$ rule of dermoscopy [45], asymmetry is given the highest weight among the four features. In the Menzies method [46], symmetry is one of the two features that rules out malignancy. In the Chaos and Clues algorithm [47], "chaos" is defined as "asymmetry of structure or colour." Asymmetry is also considered in the CASH algorithm [48] and the three-point checklist [49] guideline. During the Consensus Net Meeting on Dermoscopy [50], asymmetry was identified as one of the three criteria (the other two were atypical pigment network and blue-white structures) that are especially important in distinguishing malignant from benign pigmented skin lesions, with a good interobserver agreement between experts.

In the $\mathrm{ABCD}$ rule of dermoscopy, the lesion is bilaterally segmented by two orthogonal axes that are positioned to produce the lowest possible asymmetry score. If both axes show asymmetric contours, internal colours, and dermoscopic structures, the asymmetry score is two. If there is asymmetry on one axis only, the score is one. If asymmetry is absent with regard to both axes, the score is zero. Stolz et al. [45] highlighted that $96 \%$ of melanoma cases in their study had an asymmetry score of 2 whereas only $24 \%$ of benign lesions showed two-axis asymmetry.

Most authors focused on lesion shape (contour) to detect, measure, and quantify asymmetry. Others tried to include colour and texture in this process as well. Incorporating colour and structures are very crucial to the evaluation of lesion asymmetry since some early melanomas have symmetrical shapes [51]. In the following, we will first, briefly, review the methods that attempted to evaluate the asymmetry of shape. It will be followed by a more detailed review of methods concerned with asymmetry of colour in dermoscopy images.

5.1. Asymmetry of Shape. There have been many approaches proposed to quantify the asymmetry of lesions through analysis of lesion contour. Most of these methods try to imitate the $\mathrm{ABCD}$ rule of dermoscopy where asymmetry is quantified with respect to a symmetry axis that bisects the lesion. In automatic image analysis approaches, the symmetry axis is determined in a variety of ways, such as principal axes (moment) of inertia of the lesion shape [52]. The asymmetry is then quantified by overlapping the two halves of the lesion along the symmetry axes and dividing the nonoverlapping area differences of the two halves by the total area of the lesion [52-56].

5.2. ABCD Mimickers. Pellacani et al. [57] tried to reproduce the asymmetry evaluation approach of $\mathrm{ABCD}$ rule of dermoscopy [45] with focus on asymmetry of pigment distribution. They used principal inertia axes of lesion shape as the symmetry axes and quantified pigment distribution asymmetry based on the comparison of the difference in lightness (average grey-level values) between the two halves of the lesion along the two axes. A score of one was attributed to each axis if the grey-level differences were greater than a (empirically defined) threshold. Pellacani et al. evaluated the performance of their proposed approach by comparing it to the performance of two experienced clinicians (interobserver agreement between experts, as well as how their evaluation was combined for comparison to computer, is not described in the paper) over a set of 331 dermoscopic images (113 melanomas and 218 melanocytic nevi). They concluded "human and computer concordance was good for shape asymmetry." The human/computer concordance for pigment distribution asymmetry was lower than that observed for shape symmetry. Also, pigment distribution asymmetry was found as "the most striking parameter for diagnosis of melanoma (for both clinician and the computer)."

Seidenari et al. $[58,59]$ modified the method by Pellacani et al. In their work, a grid is laid on the image plane and aligned with the principal inertia axis. Next, colour distance between each block and its corresponding one with respect to each symmetry axis was computed. Each image block was represented by mean and standard deviation of the distribution of CIE LAB colours within the block, and the colour difference was computed using the Bhattacharyya distance [60]. To calculate a measure of symmetry, the colour differences between corresponding blocks were averaged for each axis. A score was automatically attributed to each axis: 0 if average colour distance was equal to or greater than a (empirically defined) threshold and 1 if lower. Comparison with human performance was carried out (similar to that reported in Pellacani et al.) over a set of 459 dermoscopic images. Authors reported that " $81 \%$ of symmetric/asymmetric evaluations were concordant between clinician and computer."

Ruela et al. [12] extended the approach of Seidenari et al. and studied the role of colour symmetry in automatic detection of melanoma. In addition to using the mean colour to represent each block (as originally conducted by Seidenari et al.), they used two additional descriptors: (i) unidimensional colour histograms and (ii) generalized colour moments [61]. Ruela et al. also experimented with multiple colour spaces. Moreover, instead of averaging colour difference (as in Seidenari et al.), the colour symmetry of the whole lesion is characterized by statistical properties of the colour distances (maximum, minimum, mean, and variance) which represent the distances between all symmetrical blocks.

Ruela et al. employed these features for classification of melanoma versus benign lesions on a set of 177 dermoscopy images. They experimented with a combination of different classifiers and features. The best results were achieved with the HSV colour space and the kNN classifier. Interestingly, 
the best colour descriptor was the mean colour vector (the original approach of Seidenari et al.) although the unidimensional colour histogram (computed by concatenating histograms of all colour channels) also achieved good results. This study is particularly insightful concerning their methodology and evaluation efforts. It is, however, open to criticism on three grounds: (i) lack of comparison to prior work, (ii) using a small data set, and (iii) negligence in experimenting with proposed features for discriminating between symmetric versus asymmetric lesions.

Celebi et al. [11]'s paper is yet another study that imitates the ABCD rule of dermoscopy, although they devised a new measure to quantify shape and colour asymmetry. In their approach, the image is rotated (clockwise) to align its coordinate ( $x$ and $y$ ) axes with principal inertia axis of the lesion. The lesion is then hypothetically folded around the $x$-axis and the area difference $\left(A_{x}\right)$ between the overlapping folds was taken as the amount of asymmetry on the $x$-axis. The same procedure was performed for the $y$-axis. Two asymmetry measures were calculated from $A_{x}$ and $A_{y}$ as follows:

$$
\begin{aligned}
& A_{1}=\frac{\min \left(A_{x}, A_{y}\right)}{A} \times 100 \% \\
& A_{2}=\frac{\left(A_{x}+A_{y}\right)}{A} \times 100 \% .
\end{aligned}
$$

For colour asymmetry, the same procedure is carried out except that, instead of area, pixel values were incorporated in the calculations: the sum of the absolute grey-level difference between the corresponding pixels in the two folds. The two asymmetry measures noted above $\left(A_{1}\right.$ and $\left.A_{2}\right)$ are computed for each image channel, in the RGB colour space, resulting in the total of six-coefficient (6D) colour asymmetry feature. Celebi et al. used shape and colour asymmetry features (along with other features) for classification of dermoscopy images. Experiments using support vector machine (SVM) classification, on a set of 564 images, yielded specificity and sensitivity of about $93 \%$. The paper neither evaluates the proposed asymmetry measure for detection and quantification of lesion shape/colour asymmetry nor reports the efficacy of using this feature (alone) for the main task of lesion classification (diagnosis).

Zortea et al. [62] developed an image analysis system for detection of malignant melanoma from dermoscopy images. The focus of their work was on engineering novel features, including features to quantify asymmetry of lesion shape and colouration. To that aim, they created three features that are described as follows:

(i) Asymmetry of Shape. A coordinate system is placed on lesion's center of gravity. For each axis, the difference of lesion area bisected by the axes (i.e., $\Delta S_{i}$ and $\Delta S_{j}$ ) is computed (e.g., $\left.\Delta S_{i}=\left\|\operatorname{area}\left(A_{1} \cup A_{2}\right)-\operatorname{area}\left(A_{3} \cup A_{4}\right)\right\|\right)$ and normalized by dividing by the total lesion area. The coordinate system is then rotated in steps of $10^{\circ}$. The axis with the lowest average $\Delta S$ is kept as the axis of symmetry and associated $f_{1}=\Delta S_{i}$ and $f_{2}=\Delta S_{j}$ as asymmetry of shape features. (ii) Asymmetry of Colour Intensity. The computation of this feature is similar to the calculation of shape asymmetry except that intensity values are used, instead of area, to compute $f_{3}=\Delta C_{i}$ and $f_{4}=\Delta C_{j}$. For example, $\Delta C_{i}=\Sigma_{x=0}^{255}\left\|F_{A_{1} \cup A_{2}}(x)-F_{A_{3} \cup A_{4}}(x)\right\|$, where $F(x)$ is the estimated distribution of the 256 grey-level $(x)$ values from the pixels belonging to either side of an axis, and it is computed using Gaussian kernel density estimate.

(iii) Asymmetry of Colour Shape. Zortea et al. used greylevel thresholding to segment the lesion. The center of the lesion is defined by computing the center of mass of the binary mask of the lesion. By applying different thresholds to the grey-scale values inside the lesion at percentiles $t=[0.10,0.20, \ldots, 0.90]$, different binary masks can be generated. Each would result in a different center of mass. Zortea et al. created a vector $\mathbf{v}$ whose elements were the Euclidean distances between the detected lesion centers for different threshold values. These distances were normalized by dividing by a lesion-dependent constant defined as the radius of a circle of the equivalent area as the original binary mask. Finally, average and standard deviation of $\mathbf{v}$ were used as features (i.e., $f_{5}=\mu(\mathbf{V})$ and $f_{6}=\sigma(\mathbf{V})$ ).

5.3. Classification instead of Feature Engineering. Vasconcelos et al. [63] trained different classifiers (Bayes, SVM, kNN, boosting, and Random Tree) to discriminate between symmetric and asymmetric lesions on two sets of 382 dermoscopy images (227 symmetric and 155 asymmetric) and 80 images (17 symmetric and 63 asymmetric) captured using a handheld mobile smartphone. To that aim, a total of 310 low-level features (including shape, colour, and texture) were extracted from each image. Some of these features were extracted from the entire lesion while other features were extracted from the intensity differences around the axis of inertia (like the method of Celebi et al. [11]). For example, mean and standard deviation of intensity differences were computed for each axis and each channel of RGB, HSV, and CIE LAB colour spaces, as part of colour features.

The study by Vasconcelos et al. is interesting since, unlike others who focused on feature engineering, it has mainly used low-level features and focused on classification for symmetric/asymmetric discrimination. Vasconcelos et al. also applied different feature selection techniques. The best classifier for the dermoscopic image set was the Random Forest classifier, which achieved $83 \%$ sensitivity and $89 \%$ specificity using as few as eight features. The paper, however, did not indicate which features; a reader is particularly left with the question whether the features extracted from the entire lesion or those extracted from the intensity differences around the axis of inertia were useful. These are only a few of many issues of which this study suffers. Among the other issues, the most serious is perhaps lack of comparison to prior art.

5.4. Asymmetry as Noise. Schmid-Saugeon et al. [53, 64] proposed a measure to quantify symmetry based on the mean squared error (MSE) between the original image and the 
reflected image for any potential axis of symmetry. These authors argued that any object could be decomposed to symmetric and asymmetric components. Considering the asymmetric component as symmetry noise, asymmetry can be measured using peak noise-to-signal ratio $(\mathrm{PSNR}=$ $10 \log _{10}\left(\max _{I}^{2} / \mathrm{MSE}\right)$, where $\max _{I}$ is the maximum possible pixel value of image $I$, e.g., $\max _{I}=255$ for an image represented by 8 bits per pixel). A symmetry measure can be defined [64] as

$$
\psi(\varphi, r, I)=1-\left(\frac{1}{1+\text { PSNR }}\right)
$$

where angle $\varphi$ and offset $r$ are parameters of any line (potential symmetry axis) in polar coordinates with respect to the center of lesion. The symmetry measure $\psi$ is equal to 1 only if symmetry is perfect and decreases to zero for increasing asymmetry. A lesion's axis of symmetry is any line $\left(\varphi_{l}, r_{l}\right)$ that produces $\psi\left(\varphi_{l}, r_{l}, I\right)=1$. The asymmetry score of the lesion is the one that maximizes the symmetry measure (i.e., $\left.\psi=\max _{\varphi, r} \psi(\varphi, r, I)\right)$ or in other words minimizes the mean squared error. The score and optimal parameters can be found through optimization, or by grid searching the parameter space at predefined intervals. The latter approach is practiced by the authors and visualized in what they called "the lesion symmetry map."

The MSE is computed only for pixels within the lesion. Each pixel is represented using a feature vector consisting of $u$ and $v$ (of CIE Luv colour space) components. SchmidSaugeon et al. also used Gabor texture features. SchmidSaugeon et al. did not conduct an objective experiment to evaluate the effectiveness of proposed feature to quantify asymmetry. However, the proposed asymmetry score was used as a feature to classify melanoma versus benign lesions, on a set of 100 dermoscopy images with results showing $78 \%$ sensitivity and $90 \%$ specificity. These results were achieved using a linear classifier trained in a $6 \mathrm{D}$ feature space consisting of asymmetry score using texture, colour, and shape (for shape asymmetry, instead of intensity values, MSE of the area was computed) (for each of these, asymmetry score corresponding to two axes was computed to mimic $\mathrm{ABCD}$ rule). Interestingly, texture asymmetry is reported to be the most discriminant feature although the combination of colourshape-texture produced the best results (the paper did not indicate the discriminative power of colour (asymmetry) feature).

5.5. Normalized Colour Distance. Clawson et al. [65] proposed a method to quantify colour asymmetry and also to visualize (display) it graphically. Their proposed algorithm is based on analysis of pigment distribution along radial paths from the lesion centroid to sample boundary points. For each radial path, the average greyscale value $A v_{i}$ is computed (where $i=1 \cdots N$ indexes sample points on lesion boundary). A measure is defined as Normalized Colour Distance (the naming convention is rather inappropriate since the measure does not represent the difference or distance between two colours, in a metric of interest, as it is commonly defined in colour science) $\mathrm{NDC}_{i}=A v_{i} / A_{L} \times 100$, where $A_{L}$ is the mean $A v_{i}$ for the whole lesion (i.e., $\left.A_{L}=\sum A v_{i} / N\right)$. The
NCD values were used to generate a new contour, the shape of which is indicative of pigment distribution within the lesion. For a homogeneous lesion with perfect colour symmetry, $A_{L}=A v_{i} ; \forall i$; thus $\mathrm{NCD}_{i}=100 ; \forall i$, and new contour will be a circle. Hyperpigmented areas (dark pigmentation relative to lesion average colour) will result in larger $\left(\mathrm{NCD}_{i}>\right.$ 100) values, and hypopigmented areas will result in smaller $\left(\mathrm{NCD}_{i}<100\right)$ values.

Clawson et al. derived a number of features from their proposed NCD measure and resultant symmetry contour representation. For example, since a perfectly colour symmetric lesion has a circular contour, the circularity index of the contour might be used to quantify the degree of asymmetry. The scatter plot of NCD values can also be studied for asymmetry analysis since NCD values for a perfect colour symmetric lesion would lie on a line. Deviation from the symmetry line can be used to quantify colour asymmetry.

Clawson et al. [65]'s study is one of the very few studies that has reviewed the literature; although it did not objectively compare their proposed method to the state-of-the-art one, it has cited other studies pertaining to the subject. This study is also the first to engineer a novel feature and to objectively evaluate it for detection of colour asymmetry. However, the method is tested on a set of 30 dermoscopy images with as few as eight positive samples. Therefore, we must be conservative when evaluating the effectiveness of the proposed feature. Also, interestingly, among all the proposed features, the decision tree (with the C5 algorithm) picked only one feature, named LTA/LTW in the paper, as the only significant parameter to differentiate between symmetrical and asymmetrical lesions (i.e., classification can be done by thresholding over this feature alone). The LTA/LTW feature is defined using the curve (scatter plot) of NCD values. It is computed by dividing the largest trough (the lowest point the curve sinks to) to the total area under the curve. It represents, in a way, the intensity and area of hypopigmented areas within the lesion. Hypopigmented areas represent melanoma-specific features such as regression. Therefore, one can question whether it is the colour asymmetry or presence of this feature that is being captured by the proposed method.

5.6. Reflectional Asymmetry in Histograms. Liu et al. [66, 67] proposed a method to quantify the shape and pigmentation asymmetry of skin lesions. The proposed method comprises many components, and a full review would be overlong. The description of the method's integral component, Reflectional Asymmetry in Histograms, follows. The centroid of the lesion is detected by averaging its spatial information. The lesion area is equally divided into 360 segments around the polar coordinate of its center. Each segment is represented by the area of that segment relative to the total area of the lesion. The relative area values (from 360 segments) can be plotted (e.g., from $0 \cdots 2 \pi$ ) to generate a $1 \mathrm{D}$ histogram (where each bin value corresponds to one segment value). Assuming one segment as the bilateral symmetry axis each time, the histogram is populated such that the selected segment is always at the center (in bin \#180). The asymmetry of a lesion can then be quantified by minimizing the sum of the Euclidean distances between corresponding bins from 
the left and the right halves of the histograms. In order to measure pigmentation (colour) asymmetry, instead of the relative area, one can choose, for example, (relative) average intensity values from any of $R, G$, or $B$ image channels. Instead of these image channels, Liu et al. decided to use melanin index (MI) and erythema index (EI) to "enhance the characterization of pigmentation distributions." The MI and EI are approximated from $R$ and $G$ channels according to Takiwaki et al. [68]. Moreover, instead of intensity values, Liu et al. employed a metric called global point signature (GPS) that represents "a point as a vector of scaled eigenfunctions of the Laplace-Beltrami operator computed on the object's surface [69]." Liu et al. computed the GPS representation over their proposed Lesion Pigmentation Elevation Models. The elevation models were created by mapping MI and EI values as the height information (on the $z$-axis) on the image plane. Liu et al. claimed, although not conclusive from the paper, that GPS representation "simultaneously integrates the shape and pigmentation information." The performance of the proposed asymmetry analysis was tested on 351 dermoscopy images ( 88 melanomas and 263 benign) for classification of melanoma versus benign lesions. The efficacy of the proposed method for discriminating between symmetric and asymmetric lesions remains to be tested. Interestingly, according to the authors' experiments and despite their laudable effort, the use of GPS representation, as well as employing MI and EI images, only offered a marginal improvement.

5.7. Summary and Discussion. Colour asymmetry is one of the most studied topics, compared to other colour features. Although many different techniques are proposed, most of the approaches are mimickers of the clinical ABCD rule of dermoscopy. The most successful method is probably the method of Zortea et al. [62]. Among the other techniques, the most notable are the methods of Schmid-Saugeon et al. $[53,64]$ and Clawson et al. [65] for their novelty. A list of the papers reviewed in this section is given in Table 1.

\section{Colour Variegation}

In the jargon of dermatology, variegated colouring is the term often used to describe a lesion with multiple colours. Benign lesions tend to have few colours, whereas melanomas often have many. The importance of this observation is reflected by the fact that most diagnosis guidelines [45-48] consider this feature as a sign of malignancy. In contrast, the presence of only a single colour is often enough to exclude the diagnosis of melanoma [46, 47]. Note that the dermatoscope reveals a wider range of colours than those that can be seen with the naked eye.

In clinical practice, the most common way to quantify colour variegation is to count the presence of certain colours in the lesion. In the ABCD rule of dermoscopy [45], for instance, the following six colours are considered important: light brown, dark brown, black, red, white, and blue-grey. Each of these colours in a lesion is assigned one point. The CASH algorithm [48] and Menzies's method [86] follow the same scoring principles (in the Menzies method, the colour "white" is not scored while blue and grey are considered
TABLE 1: Methods for capturing colour asymmetry.

\begin{tabular}{lcc}
\hline Authors & Year & Method \\
\hline Schmid-Saugeon et al. [64] & 2000 & Asymmetry as noise \\
Schmid-Saugeon et al. [53] & 2003 & Asymmetry as noise \\
Pellacani et al. [57] & 2006 & ABCD mimickers \\
Seidenari et al. [58] & 2006 & ABCD mimickers \\
Seidenari et al. [59] & 2006 & ABCD mimickers \\
Celebi et al. [11] & 2007 & ABCD mimickers \\
Clawson et al. [65] & 2007 & Normalized colour distance \\
Liu et al. [66] & 2011 & Reflectional asymmetry \\
Liu et al. [67] & 2012 & Reflectional asymmetry \\
Ruela et al. [12] & 2013 & ABCD mimickers \\
Vasconcelos et al. [63] & 2014 & Classification \\
Zortea et al. [62] & 2014 & ABCD mimickers \\
\hline
\end{tabular}

two colours (instead of a blue-grey colour)). Due to this diagnostic importance, the analysis of lesion colouration has been undertaken in several studies. One of the simplest approaches is to derive basic descriptive statistics from the distribution of colour primaries inside the lesion. Seidenari et al. [70, 71] divided each image into nonoverlapping blocks where each block was represented by the average colour of its contained pixels. This was followed by computing colour difference between blocks using the Euclidean distance in the RGB colour space (each colour block is compared with every other block in the image and not only to its corresponding one about the axis of symmetry). Mean, variance, and maximum colour differences were used as measures of colour distribution capturing colour variegation within the lesion. The intuitive idea behind Seidenari et al's findings is that a significant difference between RGB values means that the lesion's structure (colours/patterns) is nonhomogeneous. However, there are two drawbacks with this method. First, it is sensitive to the choice of block size. Second, larger colour differences do not necessarily mean more colour variegation. Seidenari et al. performed discriminant analysis over a set of 229 lesions and concluded that their proposed numerical parameters to capture colour variegation (i.e., mean, variance, and maximum colour block distances) are "reasonably" discriminative (higher in melanoma compared to nevi).

Abbas et al. [54] used the $k$-means clustering [87, 88] algorithm to quantize the dominant colours within a lesion. The corresponding percentage of occurrence of each colour cluster is used as features to capture colour variegation (Abbas et al. [54] are not very clear on how the feature in discussion was formed or on how it was used). A major issue with clustering is how to decide the number of clusters. Abbas et al. chose $k=6$ to imitate the ABCD rule [45]. This means the algorithm considers every lesion to be made up of six colours, even a lesion that is homogeneous with uniform colouration.

Andreassi et al. [89] described an image processing and pattern analysis software (http://www.ddax3.com/eng/index .html) which extracts "colour islands" (colour clusters within the lesion) among other image features. The details of this 
TABLE 2: Methods for capturing colour variegation.

\begin{tabular}{lccc}
\hline Authors & Year & Colour clustering & Colour distribution \\
\hline Seidenari et al. & 2005 & $\checkmark$ & $\checkmark$ \\
[70] & & & \\
Seidenari et al. & 2006 & $\checkmark$ & $\checkmark$ \\
{$[71]$} & & $\checkmark$ \\
Celebi et al. [11] & 2007 & & \\
Abbas et al. [54] & 2013 & $\checkmark$ & \\
\hline
\end{tabular}

process were not disclosed (perhaps due to patent protection). Siedenari et al. [90] noted that "colour islands [89] inside the lesion were described by polar moments of inertia (on the $x$ and the $y$ axes) of different order." From colour islands, several features were extracted [89] to represent the colour distribution of a lesion. Among these were, for example, the percentage of the island area (with respect to the lesion area) and distance from the center of the colour island to the center of the lesion. These features were studied with different agenda as these authors continued to investigate various skin diseases [55, 56, 91-104].

Celebi et al. [11] devised the feature "centroidal distances": the distance between the geometric centroid of a lesion and the brightness centroid. The former was computed from the binary lesion mask whereas the latter, the center of gravity of pixels in the lesion, was computed for each colour channel of the RGB space (and for five other colour spaces). To achieve invariance to scaling, the distance values were divided by the lesion diameter. The centroidal distance (for each colour channel) will be small if the lesion colouration is homogeneous because the brightness centroid will be close to the geometric centroid. Note that a symmetric lesion comprised of many concentric colour zones would (potentially) have the centroidal distance of zero just as a symmetric lesion made of a single shade.

6.1. Summary and Discussion. The methods described in this section can be seen as different instances of two broader approaches. Either "colour clustering" is used or "colour distribution" inside the lesion is considered to capture colour variegation (Table 2). Among these, only Umbaugh et al. specifically conducted experiments about identification of colour variegation. Other authors focused on discriminating melanoma versus benign lesions. Among those, only Seidenari et al. examined the discriminant power of their proposed feature for differentiating between melanomas and benign lesions (common and atypical nevi).

Colour variegation, although easy to define, can be difficult to identify and subjective to quantify since human capability to distinguish colours is varied and limited. Ideally, image analysis algorithms would identify this effect and measure it objectively and quantitatively to support clinical diagnosis. A major limitation of current state-of-the-art methods (Table 2) remains in that they are blind to "what" colours the lesion is comprised of. To address this defect, some researchers focused on colour classification. The next section is devoted to those methods.

\section{Colour Classification}

The objective of colour classification is to assign labels (such as colour names) to each region (or pixel) of the image using the colour information contained in that region. There are two class of approaches to achieve any classification task: generative models versus discriminative models. Generally speaking, a generative algorithm models data directly from the training samples and performs classification based on which class is most likely to generate the sample according to the model. A discriminative algorithm, on the other hand, models the differences between classes, for example, by finding decision boundaries, and simply categorizes a given sample according to those boundaries (this is an overly simplified description; for a more rigorous definition, refer to $[105,106])$.

7.1. Discriminative Models. One of the easiest methods for colour classification is to define the boundaries of each colour class in a specific colour space. Sboner et al. $[13,14,73]$ classified colours within lesions to four categories of dark brown, light brown, reddish, and whitish veil, in HSV colour space, based on this simple principle. For example, a pixel with Hue $\epsilon[13,157]$ and Saturation $\in[120,255]$ and Value $\in[0,137]$ was classified as dark brown. The boundary values were defined empirically. The success of this method is subject to the separability of colour classes in a given colour space.

Silva et al. [77] performed separability analysis of colour classes on dermoscopy images. They analysed the distribution of six different colour classes (white, black, red, blue-grey, dark, and light brown) in RGB space, using a set of 30 images, and concluded "a suitable classifier would potentially produce good results, with the exception of classes black and bluegrey that cannot be properly distinguished." Their findings, however, are limited by the fact that their training sample was confined to one annotated image (Silva et al. also considered using six annotated images for training although reportedly "this increased the dispersion of colour classes in the RGB space”).

Seidenari et al. [71, 74, 75] quantitatively evaluated their colour classifier. In their study, two clinicians independently annotated colour regions with colour names on a set of 30 dermoscopy images. From these annotated images, the average RGB values of each region were stored as an example (shade) of that colour name (class), permitting a total of 98 samples compromising a "colour palette" of six colour classes. Seidenari et al. evaluated their work on a database of 331 images (113 melanoma and 218 nevi). For each image, the two clinicians recorded the presence of each colour class. This assessment was compared with the computer's performance. The correlation between clinicians' evaluation and computer's evaluation was reported between 0.73 (for light brown) and 0.893 (for black).

Seidenari et al. also counted the number of colours in each image with results comparable to the evaluation of clinicians. They have also analysed [76] the distribution of colours in melanoma versus (atypical and common) nevi. Results showed that the number of colours in melanoma is higher than that in nevi; in particular, black, blue-grey, and 
white colours were more frequently found in melanomas. They concluded colour assessment can contribute to the distinction of melanoma, in particular from atypical nevi that share some dermoscopic features with the deadly disease. It is to be noted that labelling each pixel of each image in the nearest neighbor fashion (as practiced by Seidenari et al. and Alcon et al.) could be computationally expensive. Also, using Euclidean distance metric in RGB space for computing colour differences is a refuted practice for colour matching because RGB is not a perceptually uniform colour space. That is, colour differences in RGB do not agree consistently with human visual perception.

7.2. Generative Models. Chen et al. [72] labelled each pixel as a melanoma colour or a benign colour. Their labelling algorithm was based on histogram models. Two histograms were populated for each class (melanoma and benign) where each bin of each histogram stores the count associated with the occurrence of the bin colour in the training data for that class. The histogram counts are normalized to sum to unity, converting the histogram to (discrete) probability density function. Thus, for each class, a bin value corresponds to the likelihood of the bin colour belonging to this category. Pixels are labelled by comparing the likelihood values of their associated histogram bins. The downfall of histogram models is that classification accuracy is subject to the representativeness of training data. A pixel is labelled, for instance, as "melanoma colour" if that particular colour was observed more in melanoma training data than that of benign. Chen et al. labelled pixels as "unpopulated" if the colour was not seen in the training data (empty bin) or as "uncertain" if the likelihoods of belonging to each class were approximately equal.

Barata et al. [78] trained a Gaussian Mixture Model (GMM) for classification of five different colour classes (black, blue-grey, white, dark, and light brown) and compared its performance with that of a dermatologist. Unlike histogram models, GMMs can be made to generalize well on small amounts of training data at the cost of tuning their parameters. In their study [78], colour regions were manually segmented and labelled by a dermatologist for a training set of 27 dermoscopy images. For each colour class, small patches were randomly extracted from the corresponding segmented colour regions. The average value of HSV and LAB colour components (a 6D feature vector) from each patch is taken to represent a training sample. Barata et al. trained a GMM for each colour class using this training data. The overall performance was evaluated on a test set of 103 images (27 melanoma and $76 \mathrm{nevi}$ ). The objective was to determine presence/absence of each colour class in images. Test images were decomposed into small patches. Each patch was represented by average (HSV and LAB) colour components. Using this feature and the GMM models, the patch was labelled by comparing the likelihoods that GMMs generated. Reported results indicate average Spearman correlation of 0.7981 with the performance of human expert [78].

7.3. Summary and Discussion. All of the studies reported in this section employed colour classification to quantify the number of colours in images (lesions). As previously dis- cussed, this is to imitate clinical practices (such as $\mathrm{ABCD}$ rule) to quantify colour variegation feature of skin lesions. Among these studies, many did not directly evaluate the performance of their proposed colour classification method. Instead, they performed classification of melanoma versus benign lesions. Most studies used nearest neighbor technique in RGB colour space and define six colour classes (see Table 3). Lack of benchmarking for comparison and evaluation is a major challenge that remains to be addressed. The state of the art might be improved by incorporating and adapting existing methods in the context of image understanding for colour naming [107-110]. Colour naming is the action of assigning a linguistic colour label to image pixels and has many applications in the domain of computer vision such as for image retrieval [111], image segmentation [112], image editing [113], image description [114], pattern colourisation [115], data visualization [116], and colour constancy [117].

Among the common colours under dermoscopy, there are a few that are more specific to melanoma. For example, blue-grey or blue-whitish colour is considered a significant indicator of the disease (see 7-point checklist [118], Menzies method [86], and three-point checklist [49]). Therefore, some studies aimed at detecting certain colour and use this characteristic to classify the lesion as melanoma or benign. The next section will cover this topic.

\section{Colour Detection}

The goal is to detect specific colours that have diagnostic significance. For example, detection of blue-white veil colour or hyperpigmented areas. Colour detection is in a sense a binary colour classification. The reasons why a section is dedicated to colour detection, separated from colour classification, are twofold. Firstly, these methods do not aim to assign a class label to every pixel (or every region) in the image. They aim to identify the presence or absence of certain colours (and in some cases to localise those colours). Secondly, these methods might use features other than colour information such as texture and shape. This might seem unnecessary or even inappropriate since the task is indeed colour detection. The reader is reminded however that "colour" here is a specific dermoscopic feature and not necessarily a particular hue. For example, what dermatologist annotate as the bluewhite veil is a mixture of many different hues including blue, white, grey, and purple. The methods in this section are grouped together based on the principal approach underlying their colour detection algorithm.

8.1. Thresholding. Thresholding is one of the simplest methods of image segmentation. Ogorzałek et al. [15] used thresholding in RGB space to detect white, black, and blue-grey areas. For instance, white areas were identified as pixels which satisfied $R>110, G>R-26$, and $B>R-20$. There is no indication on how these decision rules were generated. Also, the paper does not provide any experiment to evaluate the success of colour detection. The detected colours were quantified by their area as part of a feature set for classification of skin lesions (computer-aided diagnosis). 
TABLE 3: Colour classification studies.

\begin{tabular}{llccc}
\hline Authors & Year & Method & Colour space & Number of colour classes \\
\hline Sboner et el. [13] & 2001 & Thresholding & HSV & 4 \\
Chen et al. [72] & 2003 & Histogram & RGB & 2 \\
Sboner et al. [73] & 2003 & Thresholding & HSV & 4 \\
Seidenari et al. [74] & 2003 & Nearest neighbor & RGB & 6 \\
Pellacani et al. [75] & 2004 & Nearest neighbor & RGB & 6 \\
Sboner et al. [14] & 2004 & Thresholding & HSV & 4 \\
Seidenari et al. [76] & 2005 & Nearest neighbor & RGB & 6 \\
Seidenari et al. [58] & 2007 & Nearest neighbor & RGB & 6 \\
Seidenari et al. [71] & 2006 & Nearest neighbor & RGB & 6 \\
Silva et al. [77] & 2012 & Nearest neighbor & HSV \& Lab & 6 \\
Barata et al. [78] & 2014 & Gaussian mixture & 5 \\
\hline
\end{tabular}

Pellacani et al. [71, 79] extracted dark areas in dermoscopy images. Their definition of "dark" appears to be dark brown and black pigment areas with irregular shape or asymmetric distribution that are frequently observed in melanomas. To that aim, they introduced two features: absolute dark area (ADA) and relative dark area (RDA). For ADAs, first, the mean brightness of the surrounding skin was computed as a reference brightness level. Next, the ratio between the brightness of each lesion pixel and the reference (skin's) brightness was computed. If this value was lower than a given threshold (empirically set to 0.13 ), the pixel was considered "absolutely" dark. For RDAs, the histogram of lesion brightness values were divided into four quartiles and the quantile corresponding to the lowest brightness was considered "relatively" dark. To explore the diagnostic importance of these features, statistical analysis was conducted on a set of 339 dermoscopy images (113 melanomas and 226 melanocytic nevi). For this analysis, simple numerical parameters such as region area and average intensity were computed from the detected dark areas for each lesion. Results suggested a statistically significant difference between the two classes (melanoma versus melanocytic nevi).

Sforza et al. [82] proposed an adaptive segmentation of grey areas in dermoscopy images. It seems that by "grey" the authors meant blue-grey (or blue-white) areas. The paper achieved this by thresholding on the B component of HSB colour space. The threshold values are induced "adaptively" although the paper is unclear on how this adaptive process was carried. The paper also lacks quantitative evaluation; results are shown qualitatively for only five dermoscopy images.

8.2. Decision Tree. Celebi et al. [80, 81] automatically segmented blue-white veil areas in dermoscopy images. Their approach involved pixel classification using explicit thresholding, where a trained decision tree induced the threshold values. They used a set of 105 dermoscopy images, consisting of 43 images containing sizeable blue-white veil areas with the remaining 62 free of this feature. For each image, a number of small circular regions that contain either veil or nonveil pixels were manually determined by a dermatologist and used for training. A decision tree classifier with C4.5 [119] induction algorithm was employed to classify each pixel in the training stage into two classes: veil and nonveil. Among the 18 different colour and texture features, only two features appeared in the induced decision rules: The classification was conducted by thresholding on a normalized-blue channel $(B /\{R+G+B\})$ and relative-red feature (defined as $R-\bar{R}_{s}$, where $\bar{R}_{s}$ is the mean of red channel values for healthy skin areas only). Celebi et al. further developed a second decision tree classifier to use detected blue-white veil areas for discriminating between melanoma and benign lesions. The detected veil areas were characterized using simple numerical parameters such as region area, circularity, and ellipticity measures. Experiments on a set of 545 dermoscopy images yielded $69.35 \%$ sensitivity and $89.97 \%$ specificity.

De Vita et al. $[16,83]$ detected image regions containing blue-white veil, irregular pigmentation, or regression features. To this aim, first, the lesion is segmented into homogeneous colour regions. Next, simple statistical parameters such as mean and standard deviation are extracted from HSI colour components for each region. Finally, a Logistic Model Tree (LMT) is trained to detect each colour. LMT is a supervised learning classification model that combines logistic regression and decision tree learning. De Vita et al. detected these colour features as part of their system [16] for automatic diagnosis of melanoma based on the 7-point checklist clinical guideline. They also evaluated the performance of their colour detection method over a set of 287 images (150 images were used for training and 137 for testing). It is not clear whether the test was aimed to identify (presence/absence) or to localise the colour features. Nevertheless, results were shown with average specificity and sensitivity of about $80 \%$.

8.3. Other Methods. Wadhawan et al. [84] detected bluewhite veil areas in dermoscopy images. Their method relied on a linear SVM to classify image patches to veil or nonveil. Image patches were extracted over the lesion area using a regular grid sampling. For each image patch, a feature vector was computed by concatenating histogram representation of pixel values in various colour channels of different colour spaces. They evaluated their method by performing 10-fold crossvalidation on a set of 489 dermoscopy images (163 containing 
the veil and remaining 326 free of this feature). For training, images were manually segmented and annotated by one of the authors. For testing, only presence/absence of the feature is considered. Results are reported with an average sensitivity of about $95 \%$ and an average specificity of about $70 \%$.

Lingala et al. [85] detected blue areas in dermoscopy images and further classified them to three shades of lavender and dark and light blue using fuzzy sets membership functions. Their colour detection method builds on a simple thresholding approach similar to Ogorzałek et al. [15]. A pixel is considered as "blue" if its normalized RGB values were within a certain range determined empirically (the threshold values were not reported). These blue areas were further classified into lavender and light and dark blue by thresholding their intensity value. This thresholding scheme was used to generate training data using 22 dermoscopy images. The training data was then used to determine the parameters of fuzzy set membership functions for three shades of blue. The method was evaluated over a set of 866 images (173 melanoma and 693 benign). There is no indication of how successful the colour detection was. The evaluation was conducted by classifying lesions to melanoma versus benign, by extracting simple statistical features over blue areas. Interestingly, the effect of using fuzzy set membership versus simple thresholding is evaluated and the improvement in classification is reported to be less than $0.5 \%$. Although the idea of Lingala et al. [85] is interesting, their study suffers from a number of flaws. One example is that since parameters of fuzzy sets were identical for dark and light blue, authors performed thresholding (again) over intensity channel to separate these. This could have been foreseen since intensity thresholding was used to generate training data but for fuzzy set representation, colours were represented in normalized RGB space where the intensity information is discarded.

8.4. Summary and Discussion. All the studies reported in this section can be described as using discriminative algorithms. Moreover, these studies follow the classical paradigm of supervised learning that requires extensive annotation of training images using instances of each colour feature. This is difficult (or even impossible) to be carried accurately and consistently due to the subjectivity of feature definition and poor interobserver agreements. Since ground-truth annotations were always made by an expert rather via a consensus of experts' opinions, it is hard to make outright claims about the success of these algorithms especially as all of these studies have failed to provide a comparison to other algorithms.

In terms of categorization, these works could be divided into two categories: pixel-based and region-based methods (see Table 4). Region-based methods often segment the lesion into homogeneous colour regions before further analysis. In all these cases, the emphasis is to use colour features while structural information such as texture is either ignored [15, $16,83-85]$ or found futile $[80,81]$.

\section{Colour Calibration}

For colour image processing, the fidelity of colour throughout image acquisition process is vital. Colour can dramatically change as a result of changes in imaging setup, such as varying illumination or altering acquisition device. Thus, an image processing algorithm that relies on colour information is subject to disruption. In many applications, an accurate colour calibration, therefore, appears to be necessary, to provide an image with reproducible colours, independent of the capturing system and the illuminant characteristics. This is often achieved by finding a relationship between the devicedependent output colour values of a camera (usually in RGB) and a standard colour space (such as the CIE XYZ or sRGB). The process usually involves imaging a calibrated target, often a colour checker, and then performing a least-squares regression to find a transformation matrix that maps the camera's RGB of each colour checker patch to their corresponding (standard) colour values. The measurements should be done under a known lighting system (typically D65). If the lighting cannot be controlled, then a measurement of the illuminant irradiance at each patch is needed.

Colour calibration is of double concern for dermatology since the acquisition of repeatable and high-quality images in terms of colour fidelity and resolution is essential for the comparison of time-series images during follow-up studies. However, to date, colour calibration in dermatology has been little investigated. One of the early studies that focused on colour calibration for dermatology was conducted by Haeghen et al. [120] in which a complete setup and calibration of an imaging system for use in dermatology was thoroughly described. Haeghen et al's calibration process involved estimation of camera-specific parameters (such as camera offset, colour gain, and aperture) and colour transformation matrix for standardisation. The latter was computed by acquiring images of the Macbeth colour checker chart and determining the relationship between the images and the CIE LAB values of the colour patches, acquired with a spectrophotometer. This calibration was reported to require 5-10 minutes' time of manual effort, which would remain valid for weeks of normal operation.

\section{Contrast Enhancement}

Contrast enhancement, here, refers to increasing visual discrepancy of the lesion from the normal skin surrounding it. This is often practiced as a preprocessing step aiming to improve the task of lesion segmentation. Lesion segmentation is regarded as a crucial step in dermoscopy image analysis that could affect all downstream processes to the final diagnosis. The most trivial segmentation technique is the application of grey-level thresholding methods such as Otsu's [121]. These techniques rely on the assumption that a typical dermatological image consists of two classes of pixels: the lesion and the normal skin. Therefore, the histogram of grey-level values would have two modes. Almost every method that has been proposed in the literature attempts to ensure the histogram of pixel values is bimodal and the concavity between the two modes is maximal.

Among the various proposed techniques, one of the most common practices is selecting a colour channel that maximizes the discrepancy of the lesion from the normal skin surrounding it. Ganster et al. [30], for instance, performed 
TABLE 4: Colour detection studies.

\begin{tabular}{|c|c|c|c|c|}
\hline Author & Year & Method & Approach & Target \\
\hline Pellacani et al. [79] & 2004 & Thresholding & Pixel-based & Dark areas \\
\hline Celebi et al. [80] & 2006 & Decision tree & Pixel-based & Blue-white veil \\
\hline Seidenari et al. [71] & 2006 & Thresholding & Pixel-based & Dark areas \\
\hline Celebi et al. [81] & 2008 & Decision tree & Pixel-based & Blue-white veil \\
\hline Ogorzałek et al. [15] & 2010 & Thresholding & Pixel-based & White, black, and blue-grey \\
\hline Sforza et al. [82] & 2011 & Thresholding & Pixel-based & Grey areas \\
\hline De Vita et al. [83] & 2012 & LMT & Region-based & Blue-white veil \\
\hline Wadhawan et al. [84] & 2012 & SVM & Region-based & Blue-white veil \\
\hline Fabbrocini et al. [16] & 2014 & LMT & Region-based & Blue-white veil \\
\hline Lingala et al. [85] & 2014 & Fuzzy sets & Pixel-based & Blue areas \\
\hline
\end{tabular}

thresholding over the blue channel of RGB for lesion segmentation. Madooei et al. [31, 122] found empirically that using the geometric mean of RGB channels $(\mu=\sqrt[3]{R \cdot G \cdot B})$ highlights the lesion from its surrounding and can aid segmentation based on grey-level thresholding. Schaefer et al. $[123,124]$ used the luminance $L=R+G+B$ but also applied Automatic Colour Equalization (ACE) [125] to compensate for poor contrast and lack of colour calibration. The ACE is a colour normalization technique which (in a nutshell) combines two classical techniques of max-RGB and Greyworld normalization (refer to [125] for details.). Celebi et al. [126] created a greyscale image as a weighted sum of the input RGB $\left(I=w_{1} R+w_{2} G+w_{3} B ; \sum_{i} w_{i}=1\right)$. They carried an exhaustive search over a set of possible $w_{i}$ values that maximized class separability or histogram bimodality.

Hintz-Madsen et al. [127] employed principal component analysis (PCA) of RGB data. After applying PCA, the first principal component best explains the variance in the image data. Assuming most variation occurs at the edges of the lesion, by retaining the first principal component, we shall have a greyscale of the input image that is optimal for border extraction. Madooei et al. [128] also employed PCA for contrast enhancement although the PCA was carried out in the optical density space of image data: $\log (\mathrm{RGB})$. PCA is a simple technique, easy to implement and fast to compute. On the downside, it can be speculated that PCA-based contrast enhancement would be affected by the presence of other structures such as hair or vessels in the image.

Gómez et al. [129] proposed independent histogram pursuit (IHP) that, similar to PCA, linearly transforms the original data into an uncorrelated orthogonal space. However, unlike PCA, which attempts to condense the information (variability) of measured data explained by the first component, IHP specifically aims to find projections in which the lesion and the background (healthy skin) are maximally separated. Their method is iterative and greedy (similar to that of Celebi et al. [126]). The computation of greedy techniques is more cumbersome than PCA-based methods. Gómez et al. [129] and Madooei et al. [128] are among the few who reported experimental procedure and results that showed the improvement of segmentation as a result of contrast enhancement, with a comparison to prior art. It remains to be experimentally confirmed, however, whether contrast enhancement or better segmentation offers an advantage for the task of skin lesion image analysis.

\section{Attenuation of Shading}

Shading is, to put simply, variations in image intensity due to the geometry of the scene (e.g., curvature of object surface). The effect of shading can be caused by other factors such as nonuniform illumination or as the result of a variable gain and offset in camera's sensor. In clinical images, shading is typically caused by imaging non-flat skin surfaces. In dermoscopic images, shading is usually induced by intensity falloff near the edges of the image (natural vignetting around the center of the optical axis). As a result, the image might be bright in the center and decreases in brightness as one goes to the edge of the field of view. In some other cases, the image might be darker on one side or one corner and brighter on the other side. Shading may obscure skin surface details and appear as additional features that are not intrinsic to the lesion. It can also disrupt lesion segmentation. In the literature of skin lesion analysis, most efforts are centered over postprocessing of the image to remove the shading effect.

Norton et al. [130] used adaptive histogram equalization to reduce the shading effect on the green channel before applying grey-level thresholding for segmentation of lesion. Tanaka et al. [131] subtracted each image from a background brightness that accounted for shading due to body curvature. This background image was computed by a moving average operation on each row of image data. Møllersen et al. [132] used the illumination of an empty field as a correction filter (white shading correction). In this technique, a "bright-field" image was captured before imaging the lesions by placing a "white surface" that covered camera's entire field of view in the scene. The intensity values of the image were then divided (or subtracted) from the intensity values of the bright-field image to correct the shading defect.

Madooei et al. [128] attempted to attenuate shading by normalizing the value channel of HSV colour space. They employed a photometric model to form a 1D illuminationinvariant image (from sRGB input), called the intrinsic image [133]. The intensity normalization was then induced by matching the histogram of the $\mathrm{V}$ channel to the histogram of the intrinsic image. 


\section{Conclusion}

A colour image is represented as an array of pixels, where each pixel contains numerical components (usually a triple) that defines a colour according to a colour space. Variety of colour spaces are available; they are often made for different applications (such as for display, printing, colour matching, and television broadcasting). Descriptions of different colour spaces can be found in [36].

Once the colour space is specified, colour features can be extracted from images or image regions. Some studies used pixel values directly as colour features [17, 20, 28, 134] whereas most others employed colour moments (\$3) and colour histograms $(\$ 4)$. These are primitive or low-level features that, for example, parametrize the distribution of colour value in an image. A growing trend in recent years is in developing high-level (clinically meaningful) visual features such as colour asymmetry (\$5), colour variegation (\$6), colour classification $(\$ 7)$, and colour detection $(\$ 8)$.

Among other studies, there are those that focused on “colour quantization" $[15,17,18,23,28,30,71,135,136]$ and those aimed at "colour segmentation" [132, 137-141]. Colour quantization is aimed at reducing the number of colours [88]. True-colour images typically contain thousands of colours, which makes their display, storage, transmission, and processing problematic. For this reason, colour quantization is commonly used as a preprocessing step for various graphics and image processing tasks. Most quantization methods are essentially based on data clustering algorithms. Note that colour histogram involves colour quantization.

It is worth noting that few studies [71, 75, 142-144] focused on "colour quantitation," an attempt to quantify the colour such that it can be objectively and quantitatively measured and compared. It is important to remind ourselves that colour cameras are not built for measurement of colour. They are designed to capture an image of a scene with acceptable appearance for human viewing.

When an image is captured, it may not have the optimal quality for subsequent analysis. Image enhancement is the preprocessing step that serves to compensate for the imperfections of image acquisition. Good performance of the methods at this stage not only ensures correct behaviour of the algorithms in the following stages of analysis but also relaxes the constraints on the image acquisition process. In this review paper, we also focus on those image enhancement techniques that affect the use of colour features: colour calibration $(\$ 9)$, contrast enhancement $(\$ 10)$, and shading attenuation (\$11).

\section{Competing Interests}

The authors declare that there is no conflict of interests regarding the publication of this paper.

\section{References}

[1] J. Smolle, "Automatic diagnosis," in Color Atlas of Melanocytic Lesions of the Skin, H. P. Soyer, G. Argenziano, R. HofmannWellenhof, and R. Johr, Eds., pp. 47-51, Springer, Berlin, Germany, 2007.
[2] K. Korotkov and R. Garcia, "Computerized analysis of pigmented skin lesions: a review," Artificial Intelligence in Medicine, vol. 56, no. 2, pp. 69-90, 2012.

[3] I. Maglogiannis and C. N. Doukas, "Overview of advanced computer vision systems for skin lesions characterization," IEEE Transactions on Information Technology in Biomedicine, vol. 13, no. 5, pp. 721-733, 2009.

[4] A. A. Marghoob, R. P. Braun, and J. Malvehy, "Introduction," in Atlas of Dermoscopy, A. A. Marghoob, J. Malvehy, and R. P. Braun, Eds., pp. 1-2, Informa Healthcare, 2012.

[5] M.-L. Bafounta, A. Beauchet, P. Aegerter, and P. Saiag, "Is dermoscopy (epiluminescence microscopy) useful for the diagnosis of melanoma? Results of a meta-analysis using techniques adapted to the evaluation of diagnostic tests," Archives of Dermatology, vol. 137, no. 10, pp. 1343-1350, 2001.

[6] H. Kittler, H. Pehamberger, K. Wolff, and M. Binder, "Diagnostic accuracy of dermoscopy," The Lancet Oncology, vol. 3, no. 3, pp. 159-165, 2002.

[7] C. Benvenuto-Andrade, S. W. Dusza, J. L. Hay et al., "Level of confidence in diagnosis: clinical examination versus dermoscopy examination," Dermatologic Surgery, vol. 32, no. 5, pp. 738-744, 2006.

[8] S. Q. Wang, S. W. Dusza, A. Scope, R. P. Braun, A. W. Kopf, and A. A. Marghoob, "Differences in dermoscopic images from nonpolarized dermoscope and polarized dermoscope influence the diagnostic accuracy and confidence level: a pilot study," Dermatologic Surgery, vol. 34, no. 10, pp. 1389-1395, 2008.

[9] P. Carli, V. De Giorgi, E. Crocetti et al., "Improvement of malignant/benign ratio in excised melanocytic lesions in the "dermoscopy era": a retrospective study 1997-2001," British Journal of Dermatology, vol. 150, no. 4, pp. 687-692, 2004.

[10] A. Madooei, Incorporating colour information for computeraided diagnosis of melanoma from dermoscopy images [Ph.D. thesis], Simon Fraser University, 2016.

[11] M. E. Celebi, H. A. Kingravi, B. Uddin et al., "A methodological approach to the classification of dermoscopy images," Computerized Medical Imaging and Graphics, vol. 31, no. 6, pp. 362-373, 2007.

[12] M. Ruela, C. Barata, and J. S. Marques, "What is the role of color symmetry in the detection of melanomas?" in Advances in Visual Computing, G. Bebis, R. Boyle, B. Parvin et al., Eds., vol. 8033 of Lecture Notes in Computer Science, pp. 1-10, Springer, Berlin, Germany, 2013.

[13] A. Sboner, E. Blanzieri, C. Eccher et al., "A knowledge based system for early melanoma diagnosis support," in Proceedings of the 6th IDAMA P workshop-Intelligent Data Analysis in Medicine and Pharmacology (IDAMAP '01), R. Bellazzi, B. Zupan, and X. Liu, Eds., pp. 30-35, 2001.

[14] A. Sboner, P. Bauer, G. Zumiani et al., "Clinical validation of an automated system for supporting the early diagnosis of melanoma," Skin Research and Technology, vol. 10, no. 3, pp. 184192, 2004.

[15] M. Ogorzałek, G. Surówka, L. Nowak, and C. Merkwirth, "Computational intelligence and image processing methods for applications in skin cancer diagnosis," in Biomedical Engineering Systems and Technologies: International Joint Conference, BIOSTEC 2009 Porto, Portugal, January 14-17, 2009, Revised Selected Papers, A. Fred, J. Filipe, and H. Gamboa, Eds., vol. 52 of Communications in Computer and Information Science, pp. 3-20, Springer, Berlin, Germany, 2010.

[16] G. Fabbrocini, V. D. Vita, S. Cacciapuoti et al., "Automatic diagnosis of melanoma based on the 7-point checklist," in Computer 
Vision Techniques for the Diagnosis of Skin Cancer, J. Scharcanski and M. E. Celebi, Eds., BioEngineering, pp. 71-107, Springer, Berlin, Germany, 2014.

[17] H. Iyatomi, H. Oka, M. E. Celebi et al., "An improved Internetbased melanoma screening system with dermatologist-like tumor area extraction algorithm," Computerized Medical Imaging and Graphics, vol. 32, no. 7, pp. 566-579, 2008.

[18] H. Iyatomi, H. Oka, M. E. Celebi, M. Tanaka, and K. Ogawa, "Parameterization of dermoscopic findings for the internetbased melanoma screening system," in Proceedings of the IEEE Symposium on Computational Intelligence in Image and Signal Processing (CIISP '07), pp. 189-193, Honolulu, Hawaii, USA, April 2007.

[19] I. Maglogiannis, E. Zafiropoulos, and C. Kyranoudis, "Intelligent segmentation and classification of pigmented skin lesions in dermatological images," in Advances in Artificial Intelligence, G. Antoniou, G. Potamias, C. Spyropoulos, and D. Plexousakis, Eds., vol. 3955 of Lecture Notes in Computer Science, pp. 214-223, Springer, Berlin, Germany, 2006.

[20] S. K. Tasoulis, C. N. Doukas, I. Maglogiannis, and V. P. Plagianakos, "Classification of dermatological images using advanced clustering techniques," Proceedings of the Annual International Conference of the IEEE Engineering in Medicine and Biology Society (EMBS '10), vol. Buenos Aires, Argentina, pp. 6721-6724, 2010.

[21] G. Capdehourat, A. Corez, A. Bazzano, and P. Musé, "Pigmented skin lesions classification using dermatoscopic images," in Progress in Pattern Recognition, Image Analysis, Computer Vision, and Applications, E. Bayro Corrochano and J.-O. Eklundh, Eds., vol. 5856 of Lecture Notes in Computer Science, pp. 537-544, Springer, Berlin, Germany, 2009.

[22] C. Barata, J. S. Marques, and T. Mendonc, "Bag-of-features classification model for the diagnose of melanoma in dermoscopy images using color and texture descriptors," in Image Analysis and Recognition: 10th International Conference, ICIAR 2013, Póvoa do Varzim, Portugal, June 26-28, 2013. Proceedings, M. Kamel and A. Campilho, Eds., vol. 7950 of Lecture Notes in Computer Science, pp. 547-555, Springer, Berlin, Germany, 2013.

[23] M. Binder, H. Kittler, A. Seeber, A. Steiner, H. Pehamberger, and K. Wolff, "Epiluminescence microscopy-based classification of pigmented skin lesions using computerized image analysis and an artificial neural network," Melanoma Research, vol. 8, no. 3, pp. 261-266, 1998.

[24] C. Barata, M. Ruela, T. Mendonça, and J. S. Marques, "A bagof-features approach for the classification of melanomas in dermoscopy images: the role of color and texture descriptors," in Computer Vision Techniques for the Diagnosis of Skin Cancer, J. Scharcanski and M. E. Celebi, Eds., Bio Engineering, pp. 4969, Springer, Berlin, Germany, 2014.

[25] H. Iyatomi, K.-A. Norton, M. E. Celebi, G. Schaefer, M. Tanaka, and K. Ogawa, "Classification of melanocytic skin lesions from non-melanocytic lesions," in Proceedings of the 32nd Annual International Conference of the IEEE Engineering in Medicine and Biology Society (EMBC '10), pp. 5407-5410, Buenos Aires, Argentina, September 2010.

[26] F. Xie, Y. Wu, Z. Jiang, and R. Meng, "Dermoscopy image processing for Chinese," in Computer Vision Techniques for the Diagnosis of Skin Cancer, J. Scharcanski and M. E. Celebi, Eds., BioEngineering, pp. 109-137, Springer, Berlin, Germany, 2014.

[27] N. M. Shakya, R. W. LeAnder, K. A. Hinton et al., "Discrimination of squamous cell carcinoma in situ from seborrheic keratosis by color analysis techniques requires information from scale, scale-crust and surrounding areas in dermoscopy images," Computers in Biology and Medicine, vol. 42, no. 12, pp. 1165-1169, 2012.

[28] H. Iyatomi, H. Oka, M. E. Celebi et al., "Computer-based classification of dermoscopy images of Melanocytic lesions on acral volar skin," Journal of Investigative Dermatology, vol. 128, no. 8, pp. 2049-2054, 2008.

[29] W. V. Stoecker, K. Gupta, B. Shrestha et al., "Detection of basal cell carcinoma using color and histogram measures of semitranslucent areas," Skin Research and Technology, vol. 15, no. 3, pp. 283-287, 2009.

[30] H. Ganster, A. Pinz, R. Röhrer, E. Wildling, M. Binder, and H. Kittler, "Automated melanoma recognition," IEEE Transactions on Medical Imaging, vol. 20, no. 3, pp. 233-239, 2001.

[31] A. Madooei, M. S. Drew, M. Sadeghi, and M. S. Atkins, "Intrinsic melanin and hemoglobin colour components for skin lesion malignancy detection," in Medical Image Computing and Computer-Assisted Intervention-MICCAI, N. Ayache, $\mathrm{H}$. Delingette, P. Golland, and K. Mori, Eds., vol. 7510 of Lecture Notes in Computer Science, pp. 315-322, Springer, Berlin, Germany, 2012.

[32] H. Ganster, M. Gelautz, A. Pinz et al., "Initial results of automated melanoma recognition," in Theory and Application of Image Analysis II: Selected Papers from the 9th SCIA, Scandinavian Conference on Image Analysis, pp. 343-354, Word Scientific, Singapore, 1995.

[33] M. M. Rahman and P. Bhattacharya, "An integrated and interactive decision support system for automated melanoma recognition of dermoscopic images," Computerized Medical Imaging and Graphics, vol. 34, no. 6, pp. 479-486, 2010.

[34] M. Faal, M. H. Miran Baygi, and E. Kabir, "Improving the diagnostic accuracy of dysplastic and melanoma lesions using the decision template combination method," Skin Research and Technology, vol. 19, no. 1, pp. el13-e122, 2013.

[35] W. V. Stoecker, M. Wronkiewiecz, R. Chowdhury et al., "Detection of granularity in dermoscopy images of malignant melanoma using color and texture features," Computerized Medical Imaging and Graphics, vol. 35, no. 2, pp. 144-147, 2011.

[36] T. Gevers, "Color-based retrieval," in Principles of Visual Information Retrieval, Advances in Pattern Recognition, M. S. Lew, Ed., pp. 11-49, Springer, London, UK, 2001.

[37] T. Deselaers, Features for image retrieval [Ph.D. thesis], Aachen University of Technology, 2003.

[38] H. Müller, N. Michoux, D. Bandon, and A. Geissbuhler, "A review of content-based image retrieval systems in medical applications-clinical benefits and future directions," International Journal of Medical Informatics, vol. 73, no. 1, pp. 1-23, 2004.

[39] Y. Liu, D. Zhang, G. Lu, and W.-Y. Ma, "A survey of contentbased image retrieval with high-level semantics," Pattern Recognition, vol. 40, no. 1, pp. 262-282, 2007.

[40] M. A. Stricker and M. Orengo, "Similarity of color images," in Storage and Retrieval for Image and Video Databases III, W. Niblack and R. C. Jain, Eds., vol. 2420 of Proceedings of SPIE, pp. 381-392, San Jose, Calif, USA, February 1995.

[41] R. J. Stanley, W. V. Stoecker, R. H. Moss et al., "A basis function feature-based approach for skin lesion discrimination in dermatology dermoscopy images," Skin Research and Technology, vol. 14, no. 4, pp. 425-435, 2008.

[42] N. Situ, T. Wadhawan, X. Yuan, and G. Zouridakis, "Modeling spatial relation in skin lesion images by the graph walk kernel," 
in Proceedings of the Annual International Conference of the IEEE Engineering in Medicine and Biology Society (EMB '10), pp. 6130-6133, Buenos Aires, Argentina, September 2010.

[43] M. Ruela, C. Barata, T. Mendonc, and J. S. Marques, "What is the role of color in dermoscopy analysis?" in Pattern Recognition and Image Analysis, J. M. Sanches, L. Micó, J. S. Cardoso, and J. M. Dermoscopy Analysis, Eds., vol. 7887 of Lecture Notes in Computer Science, pp. 819-826, Springer, Berlin, Germany, 2013.

[44] C. Barata, J. S. Marques, and J. Rozeira, "Evaluation of color based keypoints and features for the classification of melanomas using the bagof-features model," in Advances in Visual Computing: 9th International Symposium, ISVC 2013, Rethymnon, Crete, Greece, July 29-31, 2013. Proceedings, Part I, G. Bebis, R. Boyle, B. Parvin et al., Eds., vol. 8033 of Lecture Notes in Computer Science, pp. 40-49, Springer, Berlin, Germany, 2013.

[45] W. Stolz, A. Riemann, A. B. Cognetta et al., "ABCD rule of dermatoscopy: a new practical method for early recognition of malignant melanoma," European Journal of Dermatology, vol. 4, no. 7, pp. 521-527, 1994.

[46] S. W. Menzies, C. Ingvar, K. A. Crotty, and W. H. McCarthy, "Frequency and morphologic characteristics of invasive melanomas lacking specific surface microscopic features," Archives of Dermatology, vol. 132, no. 10, pp. 1178-1182, 1996.

[47] C. Rosendahl, A. Cameron, I. McColl, and D. Wilkinson, "Dermatoscopy in routine practice: chaos and clues," Australian Family Physician, vol. 41, no. 7, pp. 482-487, 2012.

[48] J. S. Henning, S. W. Dusza, S. Q. Wang et al., "The cash (color, architecture, symmetry, and homogeneity) algorithm for dermoscopy," Journal of the American Academy of Dermatology, vol. 56, no. 1, pp. 45-52, 2007.

[49] H. P. Soyer, G. Argenziano, I. Zalaudek et al., "Three-point checklist of dermoscopy: a new screening method for early detection of melanoma," Dermatology, vol. 208, no. 1, pp. 27-31, 2004.

[50] G. Argenziano, H. P. Soyer, S. Chimenti et al., "Dermoscopy of pigmented skin lesions: results of a consensus meeting via the internet," Journal of the American Academy of Dermatology, vol. 48, no. 5, pp. 679-693, 2003.

[51] W. Stolz, O. Braun-Falco, P. Bilek, M. Landthaler, W. H. C. Burgdorf, and A. B. Cognetta, Color Atlas of Dermatoscopy, Wiley-Blackwell, 2nd edition, 2002.

[52] I. Maglogiannis and D. I. Kosmopoulos, "Computational vision systems for the detection of malignant melanoma," Oncology Reports, vol. 15, no. 4, pp. 1027-1032, 2006.

[53] P. Schmid-Saugeon, J. Guillod, and J.-P. Thiran, "Towards a computer-aided diagnosis system for pigmented skin lesions," Computerized Medical Imaging and Graphics, vol. 27, no. 1, pp. 65-78, 2003.

[54] Q. Abbas, M. Emre Celebi, I. F. Garcia, and W. Ahmad, "Melanoma recognition framework based on expert definition of ABCD for dermoscopic images," Skin Research and Technology, vol. 19, no. 1, pp. e93-e102, 2013.

[55] P. Rubegni, A. Ferrari, G. Cevenini et al., "Differentiation between pigmented Spitz naevus and melanoma by digital dermoscopy and stepwise logistic discriminant analysis," Melanoma Research, vol. 11, no. 1, pp. 37-44, 2001.

[56] P. Rubegni, G. Cevenini, M. Burroni et al., "Automated diagnosis of pigmented skin lesions," International Journal of Cancer, vol. 101, no. 6, pp. 576-580, 2002.

[57] G. Pellacani, C. Grana, and S. Seidenari, "Algorithmic reproduction of asymmetry and border cut-off parameters according to the ABCD rule for dermoscopy," Journal of the European Academy of Dermatology and Venereology, vol. 20, no. 10, pp. 1214-1219, 2006.

[58] S. Seidenari, G. Pellacani, and C. Grana, "Early detection of melanoma by image analysis," in Bioengineering of the Skin: Skin Imaging and Analysis, K.-P. Wilhelm, P. Elsner, E. Berardesca, and H. I. Maibach, Eds., chapter 22, pp. 305-311, Informa Healthcare, CRC/Taylor \& Francis, 2007.

[59] S. Seidenari, G. Pellacani, and C. Grana, "Asymmetry in dermoscopic melanocytic lesion images: a computer description based on colour distribution," Acta Dermato-Venereologica, vol. 86, no. 2, pp. 123-128, 2006.

[60] A. Bhattacharyya, "On a measure of divergence between two statistical populations defined by their probability distributions," Bulletin of the Calcutta Mathematical Society, vol. 35, no. 1, pp. 99-109, 1943.

[61] F. Mindru, T. Moons, and L. Van Gool, "Recognizing color patterns irrespective of viewpoint and illumination," in Proceedings of the IEEE Computer Society Conference on Computer Vision and Pattern Recognition (CVPR '99), pp. 368-373, June 1999.

[62] M. Zortea, T. R. Schopf, K. Thon et al., "Performance of a dermoscopy-based computer vision system for the diagnosis of pigmented skin lesions compared with visual evaluation by experienced dermatologists," Artificial Intelligence in Medicine, vol. 60, no. 1, pp. 13-26, 2014.

[63] M. J. M. Vasconcelos, L. Rosado, and M. Ferreira, "Principal axes-based asymmetry assessment methodology for skin lesion image analysis," in Advances in Visual Computing, G. Bebis, R. Boyle, B. Parvin et al., Eds., vol. 8888 of Lecture Notes in Computer Science, pp. 21-31, Springer, Berlin, Germany, 2014.

[64] P. Schmid-Saugeon, "Symmetry axis computation for almostsymmetrical and asymmetrical objects: application to pigmented skin lesions," Medical Image Analysis, vol. 4, no. 3, pp. 269-282, 2000.

[65] K. M. Clawson, P. J. Morrow, B. W. Scotney, D. J. McKenna, and O. M. Dolan, "Computerised skin lesion surface analysis for pigment asymmetry quantification," in Proceedings of the International Machine Vision and Image Processing Conference (IMVIP '07), pp. 75-82, Kildare, Ireland, September 2007.

[66] Z. Liu, L. Smith, J. Sun, M. Smith, and R. Warr, "Biological indexes based reflectional asymmetry for classifying cutaneous lesions," in Medical Image Computing and Computer-Assisted Intervention-MICCAI, G. Fichtinger, A. Martel, and T. Peters, Eds., vol. 6893 of Lecture Notes in Computer Science, pp. 124-132, Springer, Berlin, Germany, 2011.

[67] Z. Liu, J. Sun, L. Smith, M. Smith, and R. Warr, "Distribution quantification on dermoscopy images for computer-assisted diagnosis of cutaneous melanomas," Medical \& Biological Engineering and Computing, vol. 50, no. 5, pp. 503-513, 2012.

[68] H. Takiwaki, S. Shirai, Y. Kanno, Y. Watanabe, and S. Arase, "Quantification of erythema and pigmentation using a videomicroscope and a computer," British Journal of Dermatology, vol. 131, no. 1, pp. 85-92, 1994.

[69] R. M. Rustamov, "Laplace-Beltrami eigenfunctions for deformation invariant shape representation," in Proceedings of the 5th Eurographics Symposium on Geometry Processing, pp. 225-233, Eurographics Association, 2007.

[70] S. Seidenari, G. Pellacani, and C. Grana, "Pigment distribution in melanocytic lesion images: a digital parameter to be employed for computer-aided diagnosis," Skin Research and Technology, vol. 11, no. 4, pp. 236-241, 2005. 
[71] S. Seidenari, G. Pellacani, and G. Costantino, "Automated assessment of pigment distribution and color areas for melanoma diagnosis," in Handbook of Non-Invasive Methods and the Skin, J. Serup, B. E. Jemec, and G. L. Grove, Eds., chapter 18, pp. 135-146, Informa Healthcare, CRC/Taylor \& Francis, 2nd edition, 2006.

[72] J. Chen, R. J. Stanley, R. H. Moss, and W. Van Stoecker, “Colour analysis of skin lesion regions for melanoma discrimination in clinical images," Skin Research and Technology, vol. 9, no. 2, pp. 94-104, 2003.

[73] A. Sboner, C. Eccher, E. Blanzieri et al., "A multiple classifier system for early melanoma diagnosis," Artificial Intelligence in Medicine, vol. 27, no. 1, pp. 29-44, 2003.

[74] S. Seidenari, G. Pellacani, and C. Grana, "Computer description of colours in dermoscopic melanocytic lesion images reproducing clinical assessment," British Journal of Dermatology, vol. 149, no. 3, pp. 523-529, 2003.

[75] G. Pellacani, C. Grana, and S. Seidenari, "Automated description of colours in polarized-light surface microscopy images of melanocytic lesions," Melanoma Research, vol. 14, no. 2, pp. 125130, 2004.

[76] S. Seidenari, G. Pellacani, and C. Grana, "Colors in atypical nevi: a computer description reproducing clinical assessment," Skin Research and Technology, vol. 11, no. 1, pp. 36-41, 2005.

[77] C. S. P. Silva, A. R. S. Marcal, M. A. Pereira, T. Mendona, and J. Rozeira, "Separability analysis of color classes on dermoscopic images," in Image Analysis and Recognition, A. Campilho and M. Kamel, Eds., vol. 7325 of Lecture Notes in Computer Science, pp. 268-277, Springer, Berlin, Germany, 2012.

[78] C. Barata, M. A. T. Figueiredo, M. E. Celebi, and J. S. Marques, "Color identification in dermoscopy images using Gaussian mixture models," in Proceedings of the IEEE International Conference on Acoustics, Speech and Signal Processing (ICASSP '14), pp. 3611-3615, IEEE, Florence, Italy, May 2014.

[79] G. Pellacani, C. Grana, R. Cucchiara, and S. Seidenari, "Automated extraction and description of dark areas in surface microscopy melanocytic lesion images," Dermatology, vol. 208, no. 1, pp. 21-26, 2004.

[80] M. E. Celebi, H. A. Kingravi, Y. A. Aslandogan, and W. V. Stoecker, "Detection of blue-white veil areas in dermoscopy images using machine learning techniques," in Proceedings of the Medical Imaging 2006: Image Processing, San Diego, California, USA, February 2006.

[81] M. E. Celebi, H. Iyatomi, W. V. Stoecker et al., "Automatic detection of blue-white veil and related structures in dermoscopy images," Computerized Medical Imaging and Graphics, vol. 32, no. 8, pp. 670-677, 2008.

[82] G. Sforza, G. Castellano, R. J. Stanley, W. V. Stoecker, and J. Hagerty, "Adaptive segmentation of gray areas in dermoscopy images," in Proceedings of the IEEE International Symposium on Medical Measurements and Applications (MeMeA '11), pp. 628631, Bari, Italy, May 2011.

[83] V. De Vita, G. D. L. Di Leo, G. Fabbrocini, C. Liguori, A. Paolillo, and P. Sommella, "Statistical techniques applied to the automatic diagnosis of dermoscopic images," ACTA IMEKO-The Online Journal of the International Measurement Confederation (IMEKO), vol. 1, no. 1, pp. 7-18, 2012.

[84] T. Wadhawan, R. Hu, and G. Zouridakis, "Detection of bluewhitish veil in melanoma using color descriptors," in Proceedings of the IEEE-EMBS International Conference on Biomedical and Health Informatics (BHI '12), pp. 503-506, IEEE, Hong Kong, January 2012.
[85] M. Lingala, R. Joe Stanley, R. K. Rader et al., "Fuzzy logic color detection: blue areas in melanoma dermoscopy images," Computerized Medical Imaging and Graphics, vol. 38, no. 5, pp. 403410, 2014.

[86] S. W. Menzies, C. Ingvar, and W. H. McCarthy, "A sensitivity and specificity analysis of the surface microscopy features of invasive melanoma," Melanoma Research, vol. 6, no. 1, pp. 55-62, 1996.

[87] J. A. Hartigan, Clustering Algorithms, John Wiley \& Sons, New York, NY, USA, 99th edition, 1975.

[88] M. E. Celebi, "Improving the performance of $k$-means for color quantization," Image and Vision Computing, vol. 29, no. 4, pp. 260-271, 2011.

[89] L. Andreassi, R. Perotti, M. Burroni, and G. Dell'Eva, "Computerized image analysis of pigmented lesions," Chronica Dermatologica, vol. 1, pp. 11-24, 1995.

[90] S. Seidenari, M. Burroni, G. Dell'Eva, P. Pepe, and B. Belletti, "Computerized evaluation of pigmented skin lesion images recorded by a videomicroscope: comparison between polarizing mode observation and oil/slide mode observation," Skin Research and Technology, vol. 1, no. 4, pp. 187-191, 1995.

[91] L. Andreassi, R. Perotti, P. Rubegni et al., "Digital dermoscopy analysis for the differentiation of atypical nevi and early melanoma: a new quantitative semiology," Archives of Dermatology, vol. 135, no. 12, pp. 1459-1465, 1999.

[92] P. Bauer, P. Cristofolini, S. Boi et al., "Digital epiluminescence microscopy: usefulness in the differential diagnosis of cutaneous pigmentary lesions. A statistical comparison between visual and computer inspection," Melanoma Research, vol. 10, no. 4, pp. 345-349, 2000.

[93] P. Rubegni, G. Cevenini, M. Burroni et al., "Digital dermoscopy analysis of atypical pigmented skin lesions: a stepwise logistic discriminant analysis approach," Skin Research and Technology, vol. 8, no. 4, pp. 276-281, 2002.

[94] P. Rubegni, M. Burroni, G. Cevenini et al., "Digital dermoscopy analysis and artificial neural network for the differentiation of clinically atypical pigmented skin lesions: a retrospective study," Journal of Investigative Dermatology, vol. 119, no. 2, pp. 471-474, 2002.

[95] P. Rubegni, M. Burroni, G. Dell'Eva, and L. Andreassi, “Digital dermoscopy analysis for automated diagnosis of pigmented skin lesions," Clinics in Dermatology, vol. 20, no. 3, pp. 309-312, 2002.

[96] M. Burroni, P. Rubegni, G. Dell'eva et al., "The 'common mole' from the point of view of digital dermoscopy analysis: subjective vs. objective evaluation of easy pigmented skin lesions," Journal of the European Academy of Dermatology and Venereology, vol. 17, no. 1, pp. 28-33, 2003.

[97] M. Burroni, R. Corona, G. Dell'Eva et al., "Melanoma computeraided diagnosis: reliability and feasibility study," Clinical Cancer Research, vol. 10, no. 6, pp. 1881-1886, 2004.

[98] M. Burroni, G. Dell'Eva, R. Corona et al., "Inter- and intravariability of pigmented skin lesions: could the ABCD rule be influenced by host characteristics?" Skin Research and Technology, vol. 10, no. 3, pp. 193-199, 2004.

[99] M. Burroni, P. Sbano, G. Cevenini et al., "Dysplastic naevus vs. in situ melanoma: digital dermoscopy analysis," British Journal of Dermatology, vol. 152, no. 4, pp. 679-684, 2005.

[100] M. Burroni, L. Alparone, and F. Argenti, "Comments on "a new algorithm for border description of polarized light surface microscopic images of pigmented skin lesions",' IEEE Transactions on Medical Imaging, vol. 25, no. 12, pp. 1655-1656, 2006. 
[101] U. Wollina, M. Burroni, R. Torricelli et al., "Digital dermoscopy in clinical practise: a three-centre analysis," Skin Research and Technology, vol. 13, no. 2, pp. 133-142, 2007.

[102] M. Burroni, U. Wollina, R. Torricelli et al., "Impact of digital dermoscopy analysis on the decision to follow up or to excise a pigmented skin lesion: a multicentre study," Skin Research and Technology, vol. 17, no. 4, pp. 451-460, 2011.

[103] P. Rubegni, M. Burroni, N. Nami et al., "Objective melanoma progression," Skin Research and Technology, vol. 17, no. 1, pp. 6974, 2011.

[104] P. Rubegni, G. Cevenini, N. Nami et al., "A simple scoring system for the diagnosis of palmo-plantar pigmented skin lesions by digital dermoscopy analysis," Journal of the European Academy of Dermatology and Venereology, vol. 27, no. 3, pp. e312-e319, 2013.

[105] C. M. Bishop and J. Lasserre, "Generative or discriminative? Getting the best of both worlds," Bayesian Statistics, vol. 8, pp. 3-24, 2007.

[106] A. Y. Ng and M. I. Jordan, "On Discriminative vs. Generative Classifiers: A Comparison of Logistic Regression and Naive Bayes," in Advances in Neural Information Processing Systems, T. G. Dietterich, S. Becker, and Z. Ghahramani, Eds., pp. 841-848, MIT Press, 2002.

[107] A. Mojsilović, "A computational model for color naming and describing color composition of images," IEEE Transactions on Image Processing, vol. 14, no. 5, pp. 690-699, 2005.

[108] G. Menegaz, A. Le Troter, J. Sequeira, and J. M. Boi, "A discrete model for color naming," EURASIP Journal on Advances in Signal Processing, vol. 2007, Article ID 029125, 2006.

[109] R. Benavente, M. Vanrell, and R. Baldrich, "Parametric fuzzy sets for automatic color naming," Journal of the Optical Society of America A: Optics and Image Science, and Vision, vol. 25, no. 10, pp. 2582-2593, 2008.

[110] J. van de Weijer, C. Schmid, J. Verbeek, and D. Larlus, "Learning color names for real-world applications," IEEE Transactions on Image Processing, vol. 18, no. 7, pp. 1512-1523, 2009.

[111] Y. Liu, D. Zhang, G. Lu, and W.-Y. Ma, "Region-based image retrieval with high-level semantic color names," in Proceedings of the 11th International Multimedia Modelling Conference (MMM '05), pp. 180-187, Melbourne, Australia, January 2005.

[112] T. Alarcon and O. Dalmau, "Color categorization models for color image segmentation," in Advances in Low-Level Color Image Processing, M. E. Celebi and B. Smolka, Eds., vol. 11 of Lecture Notes in Computational Vision and Biomechanics, pp. 303-327, Springer, Dordrecht, The Netherlands, 2014.

[113] J. Heer and M. Stone, "Color naming models for color selection, image editing and palette design ", in Proceedings of the ACM SIGCHI Conference on Human Factors in Computing Systems (CHI '12), pp. 1007-1016, New York, NY, USA, 2012.

[114] J. Van De Weijer and C. Schmid, "Applying color names to image description," in Proceedings of the 14th IEEE International Conference on Image Processing (ICIP '07), pp. III493-III496, September 2007.

[115] S. Lin, D. Ritchie, M. Fisher, and P. Hanrahan, "Probabilistic color-by-numbers: suggesting pattern colorizations using factor graphs," ACM Transactions on Graphics, vol. 32, no. 4, article 37, 2013.

[116] S. Lin, J. Fortuna, C. Kulkarni, M. Stone, and J. Heer, "Selecting semantically-resonant colors for data visualization," Computer Graphics Forum, vol. 32, no. 3, part 4, pp. 401-410, 2013.
[117] J. Vazquez, Colour constancy in natural images through Colour Naming and Sensor Sharpening [Ph.D. thesis], Universitat Autonomade, Barcelona, Spain, 2011.

[118] G. Argenziano, G. Fabbrocini, P. Carli, V. De Giorgi, E. Sammarco, and M. Delfino, "Epiluminescence microscopy for the diagnosis of doubtful melanocytic skin lesions: comparison of the $\mathrm{ABCD}$ rule of dermatoscopy and a new 7-point checklist based on pattern analysis," Archives of Dermatology, vol. 134, no. 12, pp. 1563-1570, 1998.

[119] J. R. Quinlan, C4.5: Programs for Machine Learning, Morgan Kaufmann, San Francisco, Calif, USA, 1993.

[120] Y. V. Haeghen, J. M. A. D. Naeyaert, I. Lemahieu, and W. Philips, "An imaging system with calibrated color image acquisition for use in dermatology," IEEE Transactions on Medical Imaging, vol. 19, no. 7, pp. 722-730, 2000.

[121] N. Otsu, "A threshold selection method from gray-level histograms," IEEE Transactions on Systems, Man, and Cybernetics, vol. 9, no. 1, pp. 62-66, 1979.

[122] A. Madooei and M. Drew, "A bioinspired color representation for dermoscopy image analysis," in Dermoscopy Image Analysis, Digital Imaging and Computer Vision, pp. 23-66, CRC Press, 2015.

[123] G. Schaefer, M. I. Rajab, M. E. Celebi, and H. Iyatomi, "Skin lesion extraction in dermoscopic images based on colour enhancement and iterative segmentation," in Proceedings of the IEEE International Conference on Image Processing (ICIP '09), pp. 3361-3364, Cairo, Egypt, November 2009.

[124] G. Schaefer, M. I. Rajab, M. E. Celebi, and H. Iyatomi, "Colour and contrast enhancement for improved skin lesion segmentation," Computerized Medical Imaging and Graphics, vol. 35, no. 2, pp. 99-104, 2011.

[125] A. Rizzi, C. Gatta, and D. Marini, "A new algorithm for unsupervised global and local color correction," Pattern Recognition Letters, vol. 24, no. 11, pp. 1663-1677, 2003.

[126] M. E. Celebi, H. Iyatomi, and G. Schaefer, "Contrast enhancement in dermoscopy images by maximizing a histogram bimodality measure," in Proceedings of the IEEE International Conference on Image Processing (ICIP '09), pp. 2601-2604, November 2009.

[127] M. Hintz-Madsen, L. K. Hansen, J. Larsen, and K. T. Drzewiecki, "A probabilistic neural network framework for detection of malignant melanoma," in Malignant Melanoma, Artificial Neural Networks in Cancer Diagnosis, Prognosis and Patient Management, pp. 141-183, CRC Press, 1999.

[128] A. Madooei, M. S. Drew, M. Sadeghi, and M. S. Atkins, "Automated pre-processing method for dermoscopic images and its application to pigmented skin lesion segmentation," in Proceedings of the 20th Color and Imaging Conference: Color Science and Engineering Systems, Technologies, and Applications (CIC '12), pp. 158-163, Los Angeles, Calif, USA, November 2012.

[129] D. D. Gómez, C. Butakoff, B. K. Ersbøll, and W. Stoecker, "Independent histogram pursuit for segmentation of skin lesions," IEEE Transactions on Biomedical Engineering, vol. 55, no. 1, pp. 157-161, 2008.

[130] K.-A. Norton, H. Iyatomi, M. E. Celebi et al., "Three-phase general border detection method for dermoscopy images using non-uniform illumination correction," Skin Research and Technology, vol. 18, no. 3, pp. 290-300, 2012.

[131] M. Tanaka, S. Gaskell, C. Edwards, and R. Marks, "Simple horizontal averaging programme enables shade correction for image analysis in psoriasis," Clinical and Experimental Dermatology, vol. 25, no. 4, pp. 323-326, 2000. 
[132] K. Møllersen, H. M. Kirchesch, T. G. Schopf, and F. Godtliebsen, "Unsupervised segmentation for digital dermoscopic images," Skin Research and Technology, vol. 16, no. 4, pp. 401-407, 2010.

[133] G. D. Finlayson, M. S. Drew, and C. Lu, "Entropy minimization for shadow removal," International Journal of Computer Vision, vol. 85, no. 1, pp. 35-57, 2009.

[134] C. S. Mendoza, C. Serrano, and B. Acha, "Pattern analysis of dermoscopic images based on FSCM color markov random fields," in Advanced Concepts for Intelligent Vision Systems, J. Blanc Talon, W. Philips, D. Popescu, and P. Scheunders, Eds., vol. 5807 of Lecture Notes in Computer Science, pp. 676-685, Springer, Berlin, Germany, 2009.

[135] M. E. Celebi and A. Zornberg, "Automated quantification of clinically significant colors in dermoscopy images and its application to skin lesion classification," IEEE Systems Journal, vol. 8, no. 3, pp. 980-984, 2014.

[136] M. E. Celebi, Q. Wen, S. Hwang, and G. Schaefer, "Color quantization of dermoscopy images using the k-means clustering algorithm," in Color Medical Image Analysis, vol. 6 of Lecture Notes in Computational Vision and Biomechanics, pp. 87-107, Springer, Dordrecht, Netherlands, 2013.

[137] P. Schmid, "Segmentation of digitized dermatoscopic images by two-dimensional color clustering," IEEE Transactions on Medical Imaging, vol. 18, no. 2, pp. 164-171, 1999.

[138] S. Umbaugh, R. Moss, W. Stoecker, and G. Hance, "Automatic color segmentation algorithms-with application to skin tumor feature identification," IEEE Engineering in Medicine and Biology Magazine, vol. 12, no. 3, pp. 75-82, 1993.

[139] R. Cucchiara, C. Grana, A. Prati, S. Seidenari, and G. Pellacani, "Building the Topological Tree by recursive FCM color clustering," in Proceedings of the IEEE International Conference on Pattern Recognition (ICPR '02), vol. 1, pp. 759-762, Quebec, Canada, 2002.

[140] S. Khak Abi, T. K. Lee, and M. S. Atkins, "Tree structured model of skin lesion growth pattern via color based cluster analysis," in Machine Learning in Medical Imaging, K. Suzuki, F. Wang, D. Shen, and P. Yan, Eds., vol. 7009 of Lecture Notes in Computer Science, pp. 291-299, Springer, Berlin, Germany, 2011.

[141] R. Melli, C. Grana, and R. Cucchiara, "Comparison of color clustering algorithms for segmentation of dermatological images," in Proceedings of the Medical Imaging 2006: Image Processing, Proceedings of SPIE, San Diego, Calif, USA, February 2006.

[142] H. Takiwaki, Y. Miyaoka, H. Kohno, and S. Arase, "Graphic analysis of the relationship between skin colour change and variations in the amounts of melanin and haemoglobin," Skin Research and Technology, vol. 8, no. 2, pp. 78-83, 2002.

[143] J. Lu, E. Kazmiercazk, J. H. Manton, and R. Sinclair, "Automatic scoring of erythema and scaling severity in psoriasis diagnosis," in Advances in Artificial Intelligence, M. Thielscher and D. Zhang, Eds., vol. 769 of Lecture Notes in Computer Science, pp. 73-84, Springer, Berlin, Germany, 2012.

[144] M. Herbin, A. Venot, J. Y. Devaux, and C. Piette, "Color quantitation through image processing in dermatology," IEEE Transactions on Medical Imaging, vol. 9, no. 3, pp. 262-269, 1990. 


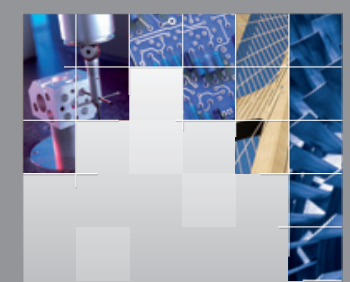

\section{Enfincering}
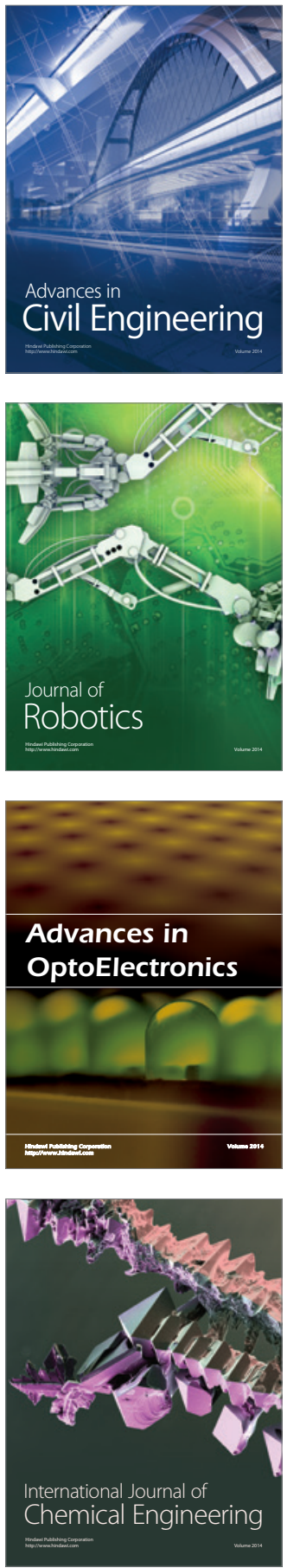

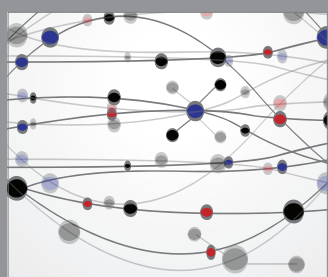

The Scientific World Journal

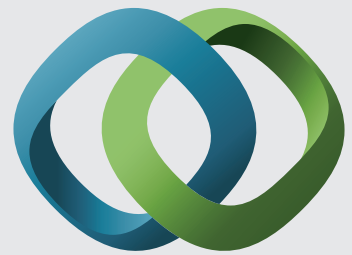

\section{Hindawi}

Submit your manuscripts at

http://www.hindawi.com
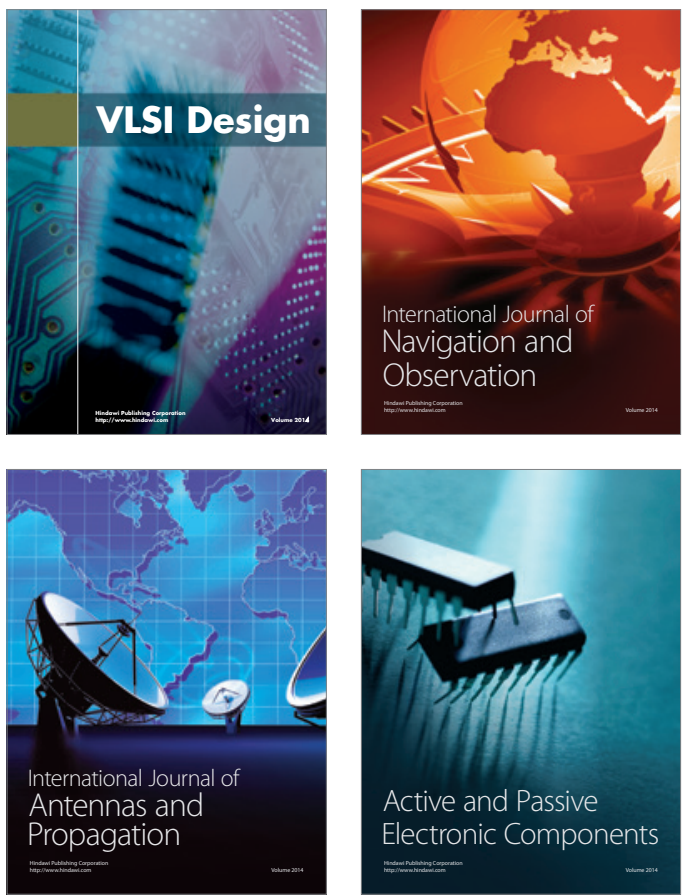
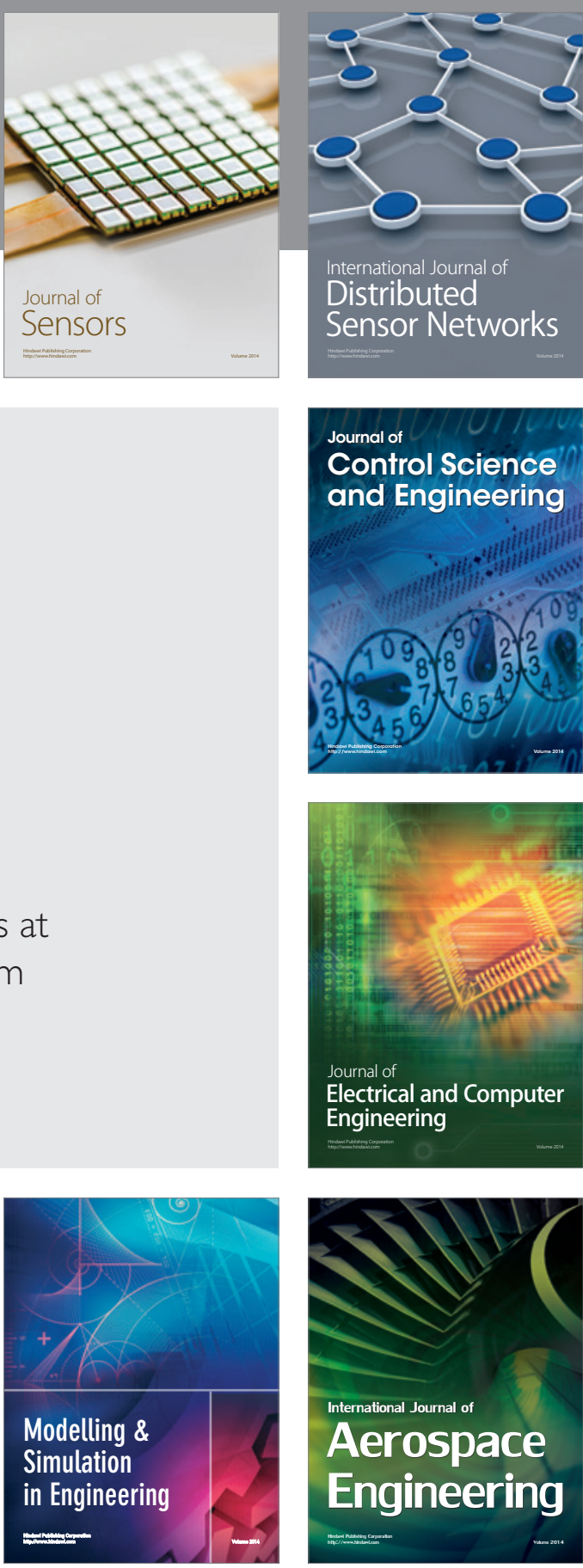

International Journal of

Distributed

Sensor Networks

Journal of

Control Science

and Engineering
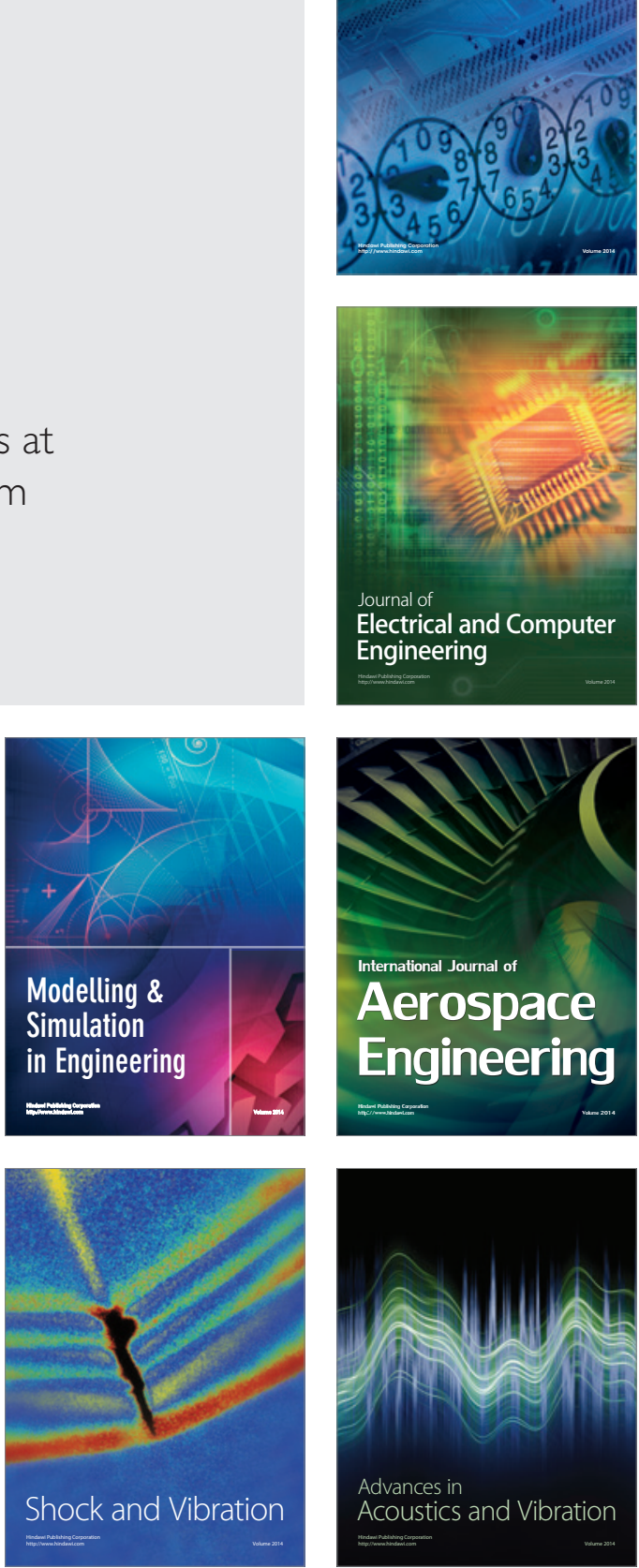